\title{
Die Bedeutung der Studierfähigkeit für den Studienerfolg im 1. Semester in Physik
}

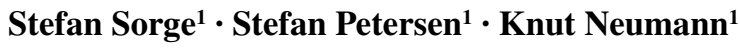

Eingegangen: 15. Dezember 2015 / Angenommen: 6. September 2016 / Online publiziert: 21. September 2016 (c) Der/die Autor(en) 2016. Dieser Artikel ist eine Open-Access-Publikation.

Zusammenfassung Das Physikstudium ist in Deutschland durch überdurchschnittlich hohe Abbruchquoten gekennzeichnet. Unklar ist jedoch, welche Faktoren ein erfolgreiches Physikstudium bedingen. Ausgehend von theoretischen Überlegungen und empirischen Befunden zum Einfluss der kognitiven und fachlichen Dimension der Studierfähigkeit auf den Studienerfolg wurden im Rahmen der hier vorgestellten Studie $N=158$ Erstsemesterstudierende an einer norddeutschen Universität befragt. Mit Hilfe einer logistischen Regression wurde der Einfluss der kognitiven und fachlichen Prädiktoren auf die Wahrscheinlichkeit des Bestehens der Abschlussklausur analysiert. Dabei konnte festgestellt werden, dass durch die Gesamtnote im Abitur und die letzte Physiknote das Bestehen von $87 \%$ der Probanden korrekt prognostiziert wird. Die Leistung im Vorwissenstest trägt zunächst nicht zur Verbesserung der Prognose bei. Betrachtet man jedoch die Note in der Abschlussklausur der erfolgreichen Probanden mit einer multiplen linearen Regression, so wird die Leistung im Vorwissenstest als einziger Prädiktor signifikant. Unterschiede im Vorwissen können somit zu einem gewissen Teil im Laufe eines Semesters kompensiert werden. Zur optimalen Förderung aller Studierenden ist es daher nötig, vorhandene Unterstützungsmaßnahmen wie Tutorien oder Vorbereitungskurse über fachspezifische Unterstützungsmaßnahmen hinaus auszubauen.

Stefan Sorge

sorge@ipn.uni-kiel.de

1 Leibniz-Institut für die Pädagogik der Naturwissenschaften und Mathematik an der Universität Kiel (IPN),

Olshausenstraße 62, 24118 Kiel, Deutschland
Schlüsselwörter Studienerfolg · Physikstudium · Kognitive Merkmale $\cdot$ Studieneingang $\cdot$ Logistische Regression

\section{The relevance of the study capacity for success in introductory physics courses}

Abstract Physics courses at German universities show above-average drop-out rates. Yet there is little empirical evidence concerning characteristics, which facilitate academic success. Based on theoretical considerations and empirical findings regarding the influence of cognitive and content-specific characteristics of the study capacity on academic success, this study surveyed $N=158$ freshmen at a German University. The influence of the predictors on the probability to succeed in the exam was evaluated using logistic regressions. It was found, that with the final school exam grade and the last physic grade the success of $87 \%$ of the participants could be classified correctly. The prior physics knowledge however didn't improve this prediction. Looking at the examination grades of the successful participants using multiple linear regressions, the prior physics knowledge was the only significant predictor. So differences in the prior knowledge could be compensated to a certain degree during the first semester. To support all students in an optimal manner the existing supporting arrangements like tutorials or preparatory courses need to be extended beyond discussing only physics content.

Keywords Academic success - Physics major - Cognitive characteristics $\cdot$ Introductory courses $\cdot$ Logistic regression 


\section{Einleitung}

Den Naturwissenschaften mangelt es an qualifiziertem Nachwuchs (Anger et al. 2014; Klemm 2015). Dies ist unter anderem dadurch bedingt, dass das Studium naturwissenschaftlicher Fächer wie Ingenieurswissenschaften, Physik oder ähnlichem als sehr anspruchsvoll gilt (Angell et al. 2004; siehe z.B. auch DIE ZEIT 05/2011: „Ingenieursstudium - Eiskalt rausgeprüft“). Wer dennoch ein naturwissenschaftliches Studium aufnimmt, muss sich zahlreichen Hürden stellen, wie die Zahlen des Deutschen Zentrums für Hochschul- und Wissenschaftsforschung (DZHW) zeigen. Heublein et al. (2014) berichten dabei in ihrer Analyse, dass in mathematisch-naturwissenschaftlichen Bachelorstudiengängen an deutschen Universitäten eine stabile Abbruchquote von $39 \%$ vorliegt. Im Vergleich dazu liegt der Durchschnitt über alle Studiengänge bei nur $33 \%$. In der Fachgruppe „Physik/Geowissenschaften“ liegt der Prozentsatz sogar noch einmal höher bei $41 \%$. Ähnliche Probleme offenbart auch der Bericht der Konferenz der Fachbereiche Physik (KFP). Dort werden Schwundquoten in der Höhe von $38 \%$ für das Wintersemester 12/13 berichtet (Matzdorf und Düchs 2013). Insgesamt schlieBen damit nur etwa 3 von 5 Studierenden ihr begonnenes physikalisches Studium auch tatsächlich ab. Das Studium der Physik ist somit sowohl durch geringe Einschreibungsquoten im Vergleich mit Studiengängen wie Jura oder Medizin (Statistisches Bundesamt 2015) als auch durch hohe Abbruchquoten gekennzeichnet. Es ist zu bezweifeln, dass damit der Bedarf an Nachwuchskräften in den Naturwissenschaften gedeckt werden kann.

Dabei sind Ursachen für den Studienabbruch bereits gut belegt (z. B. Albrecht und Nordmeier 2011; Heublein et al. 2010). Faktoren, die Studienerfolg begünstigen, sind dagegen bisher kaum bekannt. In den letzten Jahren wurden daher u. a. in der Chemie (Freyer et al. 2014) und Mathematik (Rach und Heinze 2014) Bedingungen des Studienerfolgs untersucht. Diese Untersuchungen fokussieren insbesondere auf die Studieneingangsphase, da durch den Übergang zwischen zwei Bildungsinstitutionen vermehrt Schwierigkeiten auftreten und diese Abbruchtendenzen begünstigen (Heublein et al. 2010). So werden durch den Antritt einer Erstsemesterveranstaltung die Neustudierenden erstmalig mit den Anforderungen der Universität konfrontiert. In diesem Rahmen erfolgt ein Abgleich mit den eigenen Erwartungen an das Fach, das Studium und die Rolle als Studierende selbst (Gueudet 2008; Kirsch und Vo Thi Anh 1996). Die Problematik der Anforderungen und Erwartungen gewinnt dabei zusätzliche Dynamik, da sich Einführungsveranstaltungen häufig an Studierende verschiedener Studiengänge richten. Nicht selten besuchen neben Hauptfachstudierenden und Lehramtsstudierenden der Physik auch verschiedene Profil- und Ergänzungsfächer die gleiche Veranstaltung, um die Grundlagen der (experimentellen) Physik vermittelt zu bekommen. Werden Studierende jedoch bereits in diesem Stadium des Studiums den Anforderungen nicht gerecht oder entspricht die Lehrveranstaltung nicht den Erwartungen, so kommt es gerade in dieser Phase zu Misserfolgen, die zum Studienfachwechsel oder -abbruch führen können (Heublein et al. 2010; Schiefele et al. 2007). Nur wenn Studierende erfolgreich die Hürden des ersten Semesters im Studium meistern, können sie das gesamte Studium erfolgreich abschließen. Daher bezeichnet Gainen (1995) Einführungsveranstaltungen als sogenannte „gatekeeper courses“.

Die vorliegende Studie untersucht für das Fach Physik, welche Faktoren für einen Studienerfolg nötig sind. Dazu werden zunächst theoretisch Faktoren identifiziert, welche potentiell prädiktiv für den Studienerfolg sein können. Daraus abgeleitet wird eine Studie vorgestellt und diskutiert, bei der $N=158$ Erstsemesterstudierende verschiedener physikalischer Studiengänge einer norddeutschen Universität befragt wurden. Durch die empirisch abgesicherte Bestimmung studienförderlicher Facetten wird die Ableitung von spezifischen Unterstützungsmaßnahmen möglich.

\section{Theoretischer Hintergrund}

Das Studium ist mit vielfältigen Hürden verbunden. Dies zeigt sich in der Physik an deutschlandweit überdurchschnittlich hohen Abbruchquoten (Heublein et al. 2014; Matzdorf und Düchs 2013). Die Studieneingangsphase ist dabei von besonderer Bedeutung, da mit dem Übergang vom sekundären zum tertiären Bildungssystem die Studierenden mit neuen Anforderungen konfrontiert und Anpassungsleistungen von den Studierenden verlangt werden (Gueudet 2008). Studienerfolg in dieser Phase stellt daher einen wichtigen Indikator für einen möglichen erfolgreichen Studienabschluss dar. Die Anforderungen dabei sind vielschichtig und reichen von fachinhaltlichen Problemstellungen, über die Notwendigkeit eines Nebenjobs bis zur stärkeren Eigenverantwortlichkeit im Selbststudium (Heublein et al. 2010). Um diese vielfältigen Anforderungen zu strukturieren und systematisch zu untersuchen, wird ein Modell der Studierfähigkeit und des Studienerfolgs von Heldmann (1984) verwendet, welches in weiteren Untersuchungen (Kazemzadeh et al. 1987; Konegen-Grenier 2002a; Thiel et al. 2008) weiter ausgeschärft wurde und in Abb. 1 schematisch dargestellt ist.

\section{Studienbedingungen und allgemeine Lebensbedingungen}

Als übergeordnete Rahmenbedingungen für das Studium und den Studienerfolg gelten die Studien- und allgemei- 
Abb. 1 Modell der Studierfähigkeit und des Studienerfolg (Heldmann 1984; Konegen-Grenier 2002a; Thiel et al. 2008)

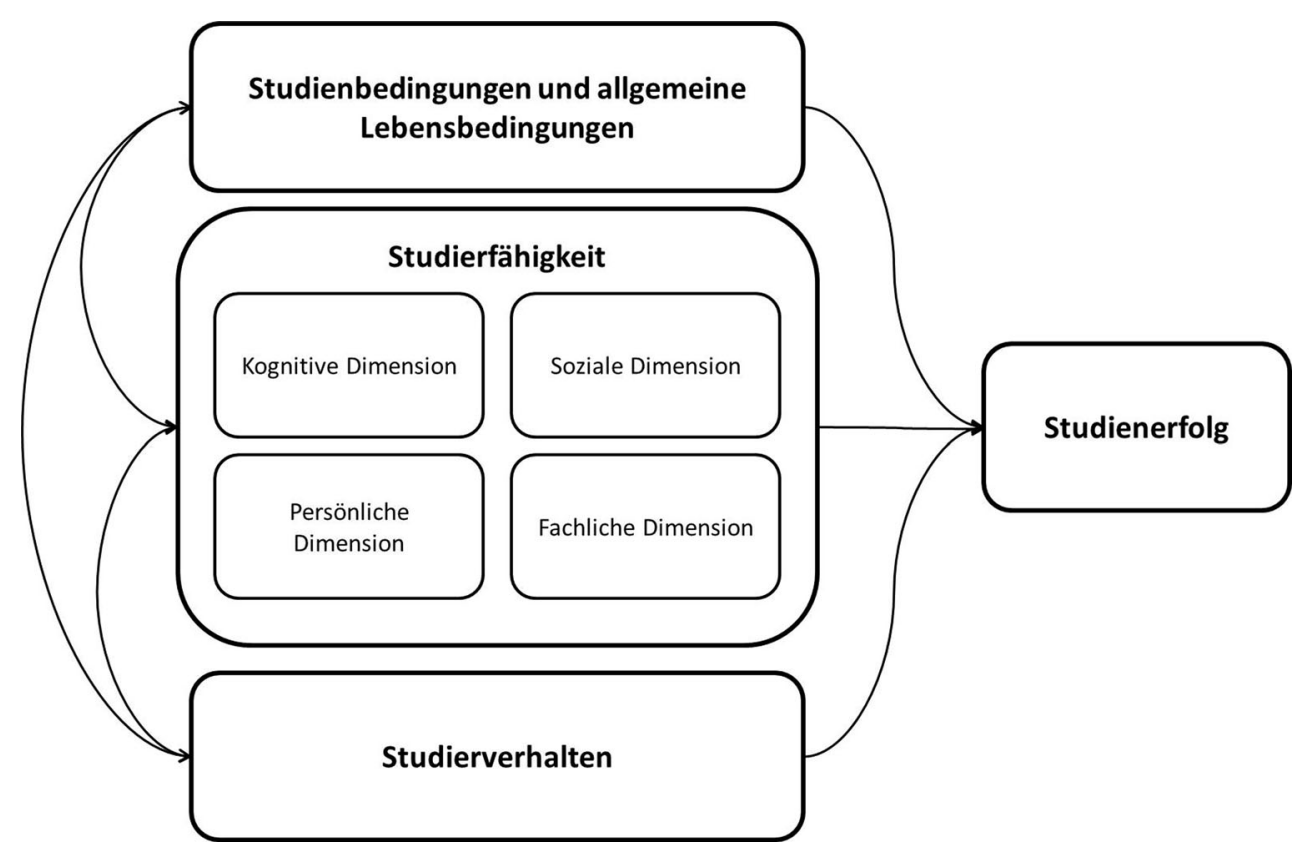

nen Lebensbedingen. Folgt man der Untersuchung von Konegen-Grenier (2002a), so schätzen die 1435 befragten Professoren die Bedeutung der Studien- und allgemeinen Lebensbedingungen auf den Studienerfolg genauso hoch ein wie den Einflussfaktor Studierfähigkeit. Während die Professoren zwar erläutern, dass die Abstimmung zwischen Studieninhalt und Prüfung, die didaktisch-methodische Aufbereitung der Vorlesungsinhalte sowie allgemeine Berufsperspektiven und soziale Unterstützungssysteme einen Einfluss auf den Studienerfolg haben, bewerten sie diese Faktoren gleichsam als weitgehend erfüllt für die Studierenden. Dieser Selbsteinschätzung widersprechen allerdings Befunde, wonach finanzielle Probleme und problematische Studienbedingungen zu den Hauptgründen für eine Exmatrikulation gehören (Albrecht und Nordmeier 2011; Heublein et al. 2010). Beide Befunde untermauern, dass die Rahmenbedingungen bei der Untersuchung des Studienerfolgs nicht vernachlässigt werden dürfen. Durch den Wechsel vom sekundären zum tertiären Bildungsbereich erfolgt zugleich eine Änderung des sozialen Umfelds für die Studierenden. Dabei erwarten Neustudierende eher die Möglichkeit zur Verwirklichung von Interessen sowie neue Kontakte. Die Konfrontation mit unerfüllten Erwartungen in Kombination mit erhöhten Leistungsanforderungen kann dann zu einem erhöhten Problemerleben führen, was schließlich im Studienabbruch enden kann (Kirsch und Vo Thi Anh 1996).

\section{Studierfähigkeit und Studierverhalten}

Um ein Studium erfolgreich bewältigen zu können, müssen Studierende verschiedenste Fähigkeiten, Fertigkeiten und
Einstellungen besitzen. Heldmann (1984) subsummiert diese vielfältigen Eingangsvoraussetzungen unter dem Begriff der Studierfähigkeit: „Die Studierfähigkeit ist dann gegeben, wenn drei Anforderungsprofile ausgeprägt vorhanden sind, nämlich allgemeine Leistungsdispositionen, eine breite Grundbildung und ein aufgabenfeldbezogener Schwerpunkt" (Heldmann und Finkenstaedt 1998). Die breit angelegte Definition folgt dabei sowohl bildungstheoretischen Überlegungen als auch einer Befragung von 11.267 Mitgliedern des deutschen Hochschulverbands (Heldmann 1984). Unter dem aufgabenfeldbezogenen Schwerpunkt versteht Heldmann einerseits die Befähigung zur begründeten Wahl eines Studiums und andererseits die wissenschaftspropädeutischen Voraussetzungen zum Umgang mit einer Wissenschaftsdisziplin (zur Wissenschaftspropädeutik siehe z. B. Trautwein und Lüdtke 2004). Weiterhin sei eine breite Grundbildung in den drei Wissenschaftsbereichen Geistes-, Gesellschafts- und Naturwissenschaften als Fundament nötig, um fachspezifische Fragestellungen in gesamtgesellschaftliche Zusammenhänge einordnen zu können (Heldmann und Finkenstaedt 1998). Schließlich unterscheidet Heldmann (1984) bei den allgemeinen Leistungsdispositionen formale, materiale und personale Bildungsvoraussetzungen, denen wiederum verschiedene Fähigkeiten und Fertigkeiten zugeordnet werden können.

Konegen-Grenier (2002a) kritisiert Heldmanns Ansatz allerdings unter dem Gesichtspunkt, dass viele einzelne Fähigkeiten und Fertigkeiten nicht trennscharf seien und $\mathrm{zu}$ den drei Bildungsvoraussetzungen auch nicht eindeutig zugeordnet werden können. Sie unterscheidet daher vier Dimensionen der Studierfähigkeit: kognitive, fachliche, persönliche und soziale Voraussetzungen. Den Angaben der 
1435 befragten Professoren zu Inhalt und Bedeutung der einzelnen Dimensionen folgend (siehe dazu auch KonegenGrenier 2002b), kommt der kognitiven Dimension die größte Bedeutung für den Studienerfolg zu. Die wichtigsten Fähigkeiten in dieser Dimension sind analytische Fähigkeiten und das Abstraktions- und Differenzierungsvermögen der Studierenden (Konegen-Grenier 2002a). Zur Bestimmung der kognitiven Voraussetzungen der Studierenden wird häufig die Abiturnote verwendet, da insbesondere die Oberstufe des Gymnasiums diese Fähigkeiten fördern und sie daher ein Maß für die erfolgreiche Bewältigung des Studiums darstellen soll (Heldmann 1984; Köller 2013). Dabei stellt die besondere Konstruktion der Abiturnote ihre große Stärke dar. Durch die Aggregation einzelner Fachnoten über einen Zeitraum von mehreren Jahren und die Beurteilung durch mehrere Lehrkräfte wird eine möglichst valide Einschätzung der Fähigkeiten eines Schülers möglich (Rindermann und Oubaid 1999; Trapmann et al. 2007). Allerdings wird auch Kritik an der Objektivität der Abiturnote geübt. So variiert die Vergabe der Abiturnote zu stark zwischen verschiedenen Bundesländern, Schulen und sogar Lehrkräften, als dass diese als alleiniges Auswahlkriterium zum Studium eingesetzt werden dürfe (Trapmann et al. 2007). Ein weiterer Kritikpunkt an der Verwendung der Abiturnote ist, dass die fachliche Dimension der Studierfähigkeit nicht hinreichend berücksichtigt wird, unter der das fachspezifische, schulische Vorwissen, das zur Bewältigung des Studiums notwendig ist, verstanden wird (Konegen-Grenier 2002a). Daraus abgeleitet sollten also eher Leistungen in studiennahen Unterrichtsfächern bei Aussagen zum Studienerfolg herangezogen werden (Rindermann und Oubaid 1999). Hierbei wäre auch denkbar, dass durch die zusätzliche Berücksichtigung der studiengangsrelevanten Fachnoten bei Verwendung der Gesamtnote im Abitur eine verbesserte Vorhersage des Studienerfolgs möglich ist, da dann sowohl allgemeine kognitive Fähigkeiten und Fertigkeiten als auch die Fachspezifik bei der Studierfähigkeit gleichermaßen Berücksichtigung finden. Um die angeführte Kritik an der Objektivität von Schulnoten zu vermeiden, kann anstelle von Fachnoten auch ein Fachwissenstest verwendet werden. Dabei sind Fachnoten und Testleistungen in Fachwissenstest zwar durchaus miteinander verknüpft, aber analog zur Abiturnote umfassen auch Fachnoten weitere Elemente, die über das reine Fachwissen hinausgehen. Solche Testleistungen wurden bisher vorwiegend bei medizinischen oder sprachlich-künstlerischen Fächern eingesetzt (Rindermann und Oubaid 1999). Im Modell der Studierfähigkeit werden allerdings keine Zusammenhänge zwischen der kognitiven und fachlichen Dimension der Studierfähigkeit diskutiert. Ebenso ist unklar, welche differenziellen Effekte fachspezifische Maße wie Fachnoten und Vorwissenstests besitzen. Insgesamt werden die kognitiven und fachlichen Voraussetzungen der Studierenden durch die Professo- ren allerdings kritisch bewertet (Konegen-Grenier 2002a). Die persönlichen Voraussetzungen, die insbesondere Persönlichkeitsmerkmale wie Interesse, Leistungsmotivation, Genauigkeit und Zielstrebigkeit umfassen, werden hingegen eher positiv eingeschätzt (Konegen-Grenier 2002a). Da es beim Übergang von der Schule zur Hochschule zu deutlichen Veränderungen der Lernumgebung (z. B. stärkere Freiwilligkeit, Vorlesung als Unterrichtsform) kommt, werden neben der kognitiven und fachlichen Dimension der Studierfähigkeit vor allem persönliche Faktoren als Prädiktoren für Studienerfolg diskutiert, die auch das Studierverhalten als wichtige dritte Säule im dargestellten Modell beeinflussen. Eine höhere Motivation führt zu einer verstärkten Einsatzbereitschaft bei Lerngelegenheiten und im Selbststudium und damit zu besseren Ergebnissen (Blömeke 2009; Schiefele und Urhahne 2000). Dabei ist anzunehmen, dass Studierende bereits ein gewisses Maß an Sachinteresse und Leistungsmotivation mitbringen, da sie sich für ein bestimmtes Fach entschieden haben. Dies steht im Einklang mit dem positiven Urteil durch Hochschullehrende in der persönlichen Dimension (Heldmann 1984; KonegenGrenier 2002a). Dabei ist jedoch zu beachten, dass motivationale und volitionale Eigenschaften einer Person von bisherigen Erfahrungen der Studierenden und von ihren kognitiven Fähigkeiten abhängen. So beeinflusst die schulische Leistungsfähigkeit bereits das Selbstkonzept der Studierenden, welches wiederum für die Lernmotivation entscheidend ist (Schiefele et al. 2003). Insgesamt sind es also vor allem kognitive Merkmale die maßgeblich den Erfolg von Studierenden in der Eingangsphase ihres Studiums beeinflussen (Konegen-Gronier 2002a). Der sozialen Dimension um Team- und Kommunikationsfähigkeit wird nur eine sehr geringe Bedeutung für den Studienerfolg zugemessen (Konegen-Grenier 2002a). Zusammenfassend kann festgehalten werden, dass umfassende kognitive Grundfähigkeiten und ein ausgeprägtes fachspezifisches Vorwissen besonders bedeutsam für Studienerfolg sind. Demgegenüber stehen jedoch auch weitere nichtkognitive Eigenschaften, die zum Teil über die Abiturnote aggregiert erfassbar werden oder maßgeblich durch sie bestimmt werden.

\section{Studienerfolg}

Studienerfolg kann anhand unterschiedlicher Kriterien bemessen werden. Diese lassen sich in subjektive, objektive und zertifizierte Studienerfolgskriterien klassifizieren. $\mathrm{Zu}$ den subjektiven Erfolgskriterien zählen vor allem die allgemeine und fachbezogene Studienzufriedenheit aber auch andere Selbsteinschätzungen wie der erlebte Kompetenzzuwachs. Diese sind insbesondere dann heranzuziehen, wenn die subjektive Verbesserung der Studienbedingungen im Fokus steht (Albrecht und Nordmeier 2011; Thiel et al. 2008). Der Wissenschaftsrat (2000) sieht das Zielkriterium 
einer universitären Ausbildung vor allem in der wissenschaftlich basierten Beschäftigungsbefähigung. Aus dieser normativen Setzung ergeben sich zwei objektive Erfolgskriterien: der Kompetenzzuwachs während des Studiums und der spätere Erfolg im Beruf durch ein Beschäftigtenverhältnis (Rindermann und Oubaid 1999). Beide objektiven Indikatoren setzen besondere Anforderungen an die methodische Herangehensweise zur Messung voraus und sind daher bisher weitgehend unberücksichtigt geblieben. Wie in allen institutionalisierten Bildungsprozessen werden Erfolge durch Abschlüsse oder Noten für die beteiligten Personen zertifiziert. Diese Erfolgsindikatoren können zwar mit subjektiven oder objektiven Erfolgen einhergehen, sind jedoch nicht identisch mit ihnen. Als grundlegende Kategorie fungiert hier erneut der erfolgreiche Studienabschluss bzw. als negativer Gegenpol der Studienabbruch (Heublein et al. 2014). In neueren Studien wird insbesondere durch die Umstellung des Studiensystems in Folge des Bologna-Prozesses die Studiendauer als ergänzendes Merkmal herangezogen (Blömeke 2009). Schließlich werden am häufigsten die Noten aus Abschluss- oder Zwischenprüfungen genutzt. Diese bieten den Vorteil, dass sie fachspezifisch angewendet werden können und qualitative Abstufungen erlauben (Rindermann und Oubaid 1999).

In den letzten Jahren wurden in den verschiedenen Fachdisziplinen empirische Arbeiten veröffentlicht, die auf eine Prognose des Studienerfolgs zu Studienbeginn abzielten. Grundlegend wurde in allen empirischen Arbeiten im Feld eine bedeutsame prognostische Validität der Gesamtnote im Abitur für den späteren Studienerfolg ermittelt. Dies konnten auch Trapmann et al. (2007) in ihrer Metaanalyse von 26 Studien mit dem Erfolgskriterium Studiennoten bestätigen. Sie fanden dabei eine mittlere gewichtete und korrigierte bivariate Produkt-Moment-Korrelation von 0,52 für Abiturnoten und Studienerfolg und identifizierten diese somit als stärksten Einzelprädiktor. In den einzelnen naturwissenschaftlichen Teildisziplinen gab es ebenfalls Bemühungen fachspezifisch geeignete Prädikatoren zu ermitteln. In einer Befragung von 165 Chemiestudierenden im ersten Semester an der Universität Duisburg-Essen konnten die Abiturnote und das fachspezifische Wissen gemessen mit 26 Multiple-Choice-Items als stärkste Prädiktoren für ein erfolgreiches Abschneiden in der Klausur am Ende des Semesters ermittelt werden (Freyer et al. 2014). Das Interesse der Studierenden zeigte nur einen geringen Einfluss, während die Effekte der Studienwahlmotivation moderierend wirkten. Einen ähnlichen Ansatz verfolgte auch die Untersuchung von Rach und Heinze (2014) in der Mathematik, welche 182 Studierende im ersten Semester untersuchten. Auch hier zeigte sich, dass sowohl gute Abiturnoten als auch ein ausgeprägtes mathematisches Vorwissen im Bereich Analysis wichtige Voraussetzungen für das erfolgreiche Bestehen der Klausur zur Analysis-Ein- führungsvorlesung an einer Universität sind. In weiteren Analysen zeigte sich jedoch auch, dass Interesse und Motivation zwar keinen direkten Einfluss auf den Studienerfolg besitzen, aber verschiedene Lerntypen unterschiedliche Erfolgschancen besaßen (Rach und Heinze 2013). Zusammengefasst lässt sich also festhalten, dass Studien in artverwandten naturwissenschaftlichen Studiengängen die kognitiven Eingangsmerkmale Abiturnote und fachspezifisches Vorwissen als Haupteinflussfaktoren auf den Studienerfolg identifizieren konnten. Die Ergebnisse im Bereich motivationaler Faktoren hingegen sind nicht eindeutig und es scheint kein direkter Einfluss vorzuliegen (Freyer et al. 2014; Rach und Heinze 2014). Ob Befunde aus anderen Studiengängen übertragbar sind, ist jedoch unklar. Dies liegt insbesondere an der thematischen Besonderheit, die Einführungskurse an Universitäten auszeichnet. Die Physik unterscheidet sich von anderen mathematisch-naturwissenschaftlichen Studienfächern, da in den ersten zwei Semestern wesentliche Inhalte der Schulphysik aufgegriffen und vertieft werden, während in anderen naturwissenschaftlichen Vorlesungen bereits stärkere Spezialisierungen vorgenommen werden (z. B. Analysis oder Anorganische Chemie).

Analoge Studien für den Fachbereich Physik liegen bisher nicht vor. Es existieren allerdings auch hier erste Erkenntnisse. So untersuchten Albrecht und Nordmeier (2011) für Studienanfänger das (Miss-)Erfolgskriterium Studienabbruch. Sie konnten für 140 Studierende im zweiten Semester feststellen, dass die Abiturnote, das Studienwahlmotiv, sowie die Studienbedingungen (z.B. wahrgenommene Betreuung und Unterstützung) die Abbruchtendenz signifikant beeinflussen. Diese Erkenntnisse decken sich mit Befunden aus den USA, die vorwiegend kognitive Merkmale als förderlich für den Verbleib in einem naturwissenschaftlichem Studium identifizierten (Shaw und Barbuti 2010). Hazari et al. (2007) konnten in einer umfassenden Studie in den USA ebenfalls zeigen, dass dort der akademische Hintergrund (u. a. Noten in Englisch, Mathematik und Science, SAT-Score in Mathe) der wichtigste Prädiktor für ein positives Ergebnis bei Einführungskursen der Physik ist. Auch dort erscheint die fachliche Vorbereitung in der High-School zusätzliche Bedeutung zu besitzen, die jedoch nur über retrospektive Angaben der Behandlungsdauer einzelner Themen erfasst wurde. Es zeigt sich also auch hier, dass die kognitiven und fachlichen Fähigkeiten der Studierenden entscheidende Faktoren für Studienerfolg im ersten Semester darstellen. Auffallend ist jedoch auch, dass bisherige Studien vorwiegend einzelne Studienerfolgskriterien betrachtet haben und keine Vergleiche vorgenommen wurden. Eine gleichzeitige Berücksichtigung der kognitiven und fachlichen Fähigkeiten, ihre gegenseitige Beeinflussung sowie ihre Prädiktivität 
im Hinblick auf verschiedene Studienerfolgskriterien im ersten Semester in Physik stehen daher noch aus.

\section{Fragestellung und Hypothesen}

Die vorliegende Forschungsarbeit widmet sich der Fragestellung, inwiefern kognitive und fachliche Eingangsvoraussetzungen für den Studienerfolg im ersten Semester für ein Studium der Physik entscheidend sind. Zu Beginn eines Physikstudiums steht dabei zumeist eine experimentell orientierte Lehrveranstaltung, die grundlegende Inhalte in Mechanik und Thermodynamik präsentiert und in Übungen vertieft. Folgt man dem idealtypischen Aufbau eines Lehramtsstudiums der Physik nach den Vorschlägen der Deutschen Physikalischen Gesellschaft (DPG) (2014), so werden in der Physik 1-Veranstaltung Themen wie Kinematik, Newtonsche Gesetze, Arbeit, Energie, Schwingungen, Rotation, Gase und Flüssigkeiten im Bereich der Mechanik behandelt. Zentrale Inhalte der Thermodynamik sind die Hauptsätze der Thermodynamik, die Zustandsgleichung für ideale Gase, Kreisprozesse und erste statistische Verteilungen. Die zugeordneten Übungen dienen der Wiederholung, Vertiefung und Anwendung des Lehrstoffes der Vorlesung. Neben der grundlegenden Ausbildung in der Physik 1-Veranstaltungen ergeben sich unterschiedliche weitere Schwerpunkte für Fachphysik- oder Lehramtsstudierende wie beispielsweise Einführung in mathematische Methoden oder erste eigene experimentelle Erfahrungen in Grundpraktika. Die vielfältigen Einflüsse der Studien- und Lebensbedingungen lassen sich dabei allerdings nur schwer in einem kontrolliertem Forschungsdesign in den Blick nehmen. Der Fokus dieses Forschungsvorhabens liegt daher auf der Stu- dierfähigkeit und hält dementsprechend die Studienbedingungen weitgehend konstant.

Der Studienerfolg zu Beginn des Physikstudiums stellt das erfolgreiche Bestehen der ersten Klausur dar. Das Überwinden dieser Hürde spiegelt am deutlichsten den Gedanken eines ,gatekeepers“ wider. Die Güte des Bestehens bestimmt durch die Note in der Abschlussklausur erscheint darüber hinaus ein weiteres Studienerfolgskriterium mit Einfluss für das weitere Studium (vgl. Abb. 2).

Als zentrale Voraussetzung für den Studienerfolg gelten die kognitive und die fachliche Dimension der Studierfähigkeit. Durch die Gesamtnote im Abitur werden zentrale Fähigkeiten und Fertigkeiten der kognitiven Dimension der Studierfähigkeit erfasst (Rindermann und Oubaid 1999; Trapmann et al. 2007). Daher ergibt sich folgende Hypothese:

1. Die Gesamtnote im Abitur als Maß für die kognitive Dimension der Studierfähigkeit ist der stärkste Prädiktor für das Bestehen und die Note in der Abschlussklausur im ersten Semester eines Physikstudiums.

Darüber hinaus sind neben Elementen der kognitiven Dimension wie z. B. analytische Fähigkeiten auch fachspezifische Fähigkeiten und Fertigkeiten bedeutsam für eine ausgeprägte Studierfähigkeit (Konegen-Grenier 2002a). Elemente der fachlichen Dimension stehen dabei zwar im $\mathrm{Zu}$ sammenhang mit der kognitiven Dimension (vgl. Abb. 2), fokussieren aber stärker auf die Spezifik des Studiengangs. Analog zur Gesamtnote im Abitur umfasst auch die letzte Physiknote verschiedene Leistungsmerkmale der fachlichen Dimension, wobei das fachspezifische Vorwissen nur teilweise Berücksichtigung findet. Dieses kann gesondert über die Leistung in einem Vorwissenstest erfasst werden. Auf Grundlage der Ergebnisse in anderen mathematisch-
Abb. 2 Untersuchungsmodell zum Zusammenhang der Elemente der Studierfähigkeit und des Studienerfolgs

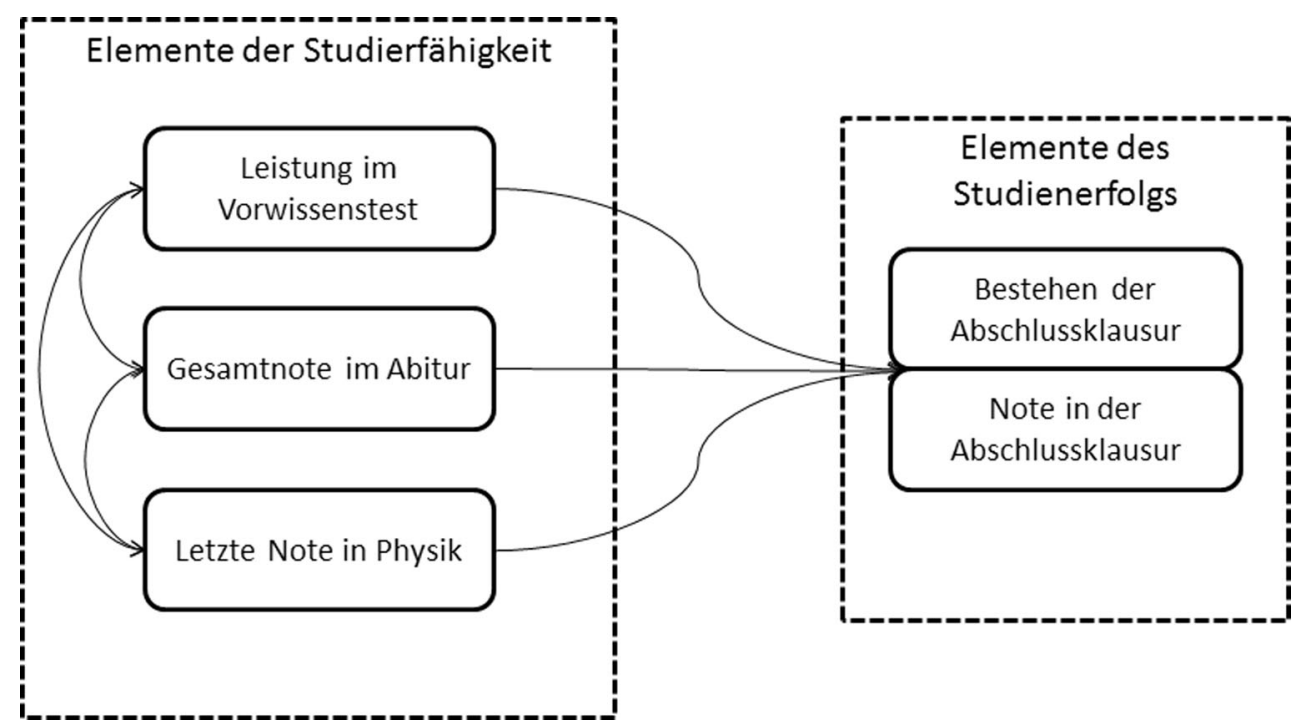


naturwissenschaftlichen Studiengängen (Freyer et al. 2014; Rach und Heinze 2014), kann daher folgende Hypothese formuliert werden:

2. Die Berücksichtigung der Leistung in einem Vorwissenstest und der letzten Note in Physik führt zu einer Verbesserung der Prognosekraft für das Bestehen und die Note der Abschlussklausur unter Berücksichtigung der Abiturnote.

Die differenziellen Zusammenhangsmuster zwischen Elementen der Studierfähigkeit und des Studienerfolgs werden in der folgenden Studie untersucht.

\section{Methode}

\section{Design}

Ziel der vorliegenden Studie ist zu untersuchen, inwiefern kognitive und fachliche Eingangsmerkmale der Studierenden wie die Leistung im Vorwissenstest, die Gesamtnote im Abitur und die letzte Physiknote den Studienerfolg im ersten Semester vorhersagen. Dazu wurden Daten von $N=$ 158 Studierenden im Wintersemester 2014/2015, die die Einführungsvorlesung der Physik einer mittelgroßen norddeutschen Universität besuchten, erhoben und ausgewertet. Die Befragung in der ersten Sitzung der Lehrveranstaltung umfasste neben demographischen Angaben wie Alter, Geschlecht, Abiturnote u. a. auch die Abfrage, ob das Studienfach ein Wunschfach war und einen Test, um das physikalische Vorwissen aus der Schule zu erfassen. Zur Bearbeitung des Fragebogens standen 45 min zur Verfügung. Die vollständigen Angaben bei einzelnen Items variiert dabei von $100 \%$ (Vorwissenstest) bis $58 \%$ (letzte Physiknote), wobei für den demographischen Hintergrund der Studierenden auch Angaben aus einer Befragung im Vorkurs ergänzt wurden.

Der Aufbau der Veranstaltung entspricht weitgehend dem idealtypischen Standard, der beispielsweise von der DPG (2014) gefordert wird. So gliedert sich das Modul „Physik 1“ in eine Vorlesung mit vier Semesterwochenstunden und zwei Semesterwochenstunden Übung. Auch die Lehrinhalte laut Modulhandbuch sind im Bereich der Mechanik mit Koordinaten- und Bezugssysteme, Kinematik, spezielle Relativitätstheorie, Dynamik und Newtonsche Gesetze, Schwingungen, Hydrostatik und -dynamik sowie Aerodynamik weitgehend idealtypisch. Gleiches gilt für den Bereich der Thermodynamik mit den Lehrinhalten Wärmelehre, Gasgesetze und Grundzügen der statistischen Thermodynamik. Mit Blick auf die Lernziele wird formuliert, dass die Studierende grundlegende Vorgänge der Mechanik und Wärmelehre an Hand von Demonstrations- experimenten kennen und die mathematische Beschreibung der physikalischen Gesetze beherrschen sollen.

Die Studierenden wurden im Anschluss an die Abschlussklausur gebeten, ihren bei der ersten Testung verwendeten persönlichen Code erneut anzugeben, um die zuvor aufgenommenen Daten mit dem Klausurergebnis in Verbindung bringen zu können. Die Abschlussnote am Ende des ersten Semesters diente als Maß für den zertifizierten Studienerfolg. Die Klausur traten zum ersten Termin 141 Studierende an. 60 davon stimmten zu, ihre Klausurnote zur Verfügung zu stellen, was einer Rücklaufquote von etwa $43 \%$ entspricht. An dem zweiten Prüfungstermin nahmen 63 Studierende teil, wovon 52\% ihre Abschlussnote zur Verfügung stellten. Zur Analyse wurden die Daten beider Zeitpunkte verwendet. Lagen sowohl Ergebnisse vom ersten als auch vom zweiten Klausurzeitpunkt vor, so wurde die bessere Note berücksichtigt, da die Studierenden schließlich den ,gatekeeper“ überwunden hatten. Insgesamt konnten somit 77 Klausurergebnisse berücksichtigt werden.

\section{Stichprobe}

An der ersten Vorlesung im Semester und somit der ersten Erhebung nahmen insgesamt $N=239$ Studierende teil, wobei $25 \%$ davon weiblich waren. Das Durchschnittsalter betrug 20,8 Jahre ( $S D=3,2$ Jahre). Die Teilnehmer setzten sich aus fünf verschiedenen Studiengruppen zusammen: Bachelor Physik (23\%), Bachelor Materialwissenschaften (23\%), Bachelor Physik des Erdsystems (21\%), Lehramtsstudierende der Physik (23\%) und verschiedene Nebenfachstudierende (5\%). Der Studiengang Physik des Erdsystems stellt eine Besonderheit des Standorts dar. Der Schwerpunkt der Ausbildung liegt hier auf der Verknüpfung der Fachbereiche Geophysik, Ozeanographie und Meteorologie. Das Studium der Materialwissenschaften kombiniert Elemente des Physikstudiums und der Ingenieurswissenschaften mit der Beschäftigung mit speziellen Werkstoffen. Alle Studierendengruppen müssen jedoch die gleiche Vorlesung besuchen, um grundlegende Kenntnisse der Mechanik und Thermodynamik zu erlangen. Die verschiedenen $\mathrm{Ne}$ benfachstudierenden stellen eine stark heterogene Studiengruppe dar, die sich aus Bachelorstudierenden der Fächer Chemie, Mathematik und Informatik zusammensetzt. Auf Grund der schweren Vergleichbarkeit dieser Studierenden mit den übrigen, klarer definierten Studiengängen, werden diese in der weiteren Analyse nicht berücksichtigt. Hinzu kommt, dass die Studierenden der Materialwissenschaften eine eigene Übung angeboten bekommen und auch eine andere Klausur schreiben. Da diese Studierenden also andere Kriterien für einen Studienerfolg erfüllen müssen, wurden sie bei der Auswertung ebenfalls nicht berücksichtigt. Weiterhin machten 12 Studierende keine Angaben zu ihrem 
Studiengang. Die finale Analysestichprobe umfasst somit $N=158$ Studierende der Fächer Bachelor Physik, Physik des Erdsystems und Lehramt Physik. $27 \%$ der untersuchten Probanden waren weiblich und das mittlere Alter betrug 20,6 Jahre $(S D=2,6)$. Die demographischen Daten der Analysestichprobe unterscheiden sich somit nur geringfügig von denen der Ausgangsstichprobe. Zwischen den verschiedenen Studiengängen zeigen sich signifikante Unterschiede in der Geschlechterverteilung $\left(\chi^{2}(2, N=158)=\right.$ $7,19, p<0,05)$. So variiert der Anteil weiblicher Studierender von $15 \%$ bei den Studierenden des Bachelor Physik bis zu $37 \%$ im Studiengang Bachelor Physik des Erdsystems. Weiterhin gaben $87 \%$ der Befragten an im 1. Fachsemester zu studieren, so dass nur eine geringe Anzahl von Studierenden zu erwarten ist, die die Vorlesung eventuell wiederholen. Im Vergleich mit den offiziell gemeldeten Studierendendaten der Studiengänge Bachelor Physik, Physik des Erdsystems und Lehramt Physik zeigt sich, dass sich etwa 30 Studierende mehr angemeldet als tatsächlich an der ersten Vorlesung teilgenommen haben. Es ist allerdings davon auszugehen, dass die tatsächliche Präsenz in der Veranstaltung ein besserer Indikator für die Teilnahme an der Lehrveranstaltung ist. Insgesamt kann somit von einem hohen Ausschöpfungsgrad aller Studierenden der drei Studienfächer an der Universität ausgegangen werden.

\section{Instrumente}

Der Fragebogen der ersten Erhebung bestand aus zwei Teilen. Der erste Teil beinhaltete acht Fragen zum demographischen und schulischen Hintergrund. Diese umfassten das Geschlecht, das Alter, Informationen zum Studiengang und Informationen zur bisherigen schulischen Bildung inklusive der Gesamtnote im Abitur (Schulnoten 1-6) und der letzten Physiknote (Punktesystem der Oberstufe 0-15). Außerdem sollten die Studierenden angeben, ob das gewählte Studienfach dem Wunschfach entspricht. In Anlehnung an Freyer et al. (2014) sollen über diese Information, die mit „Ja, das studierte Fach ist mein Wunschfach“, „Nein, ich hätte lieber etwas anderes studiert" oder "Teils ja, teils nein“" beantwortet werden konnte, Aussagen zur Studienwahlmotivation möglich werden.

Für den zweiten Teil wurde ein spezieller Vorwissenstest der Physik für die Studierenden entwickelt. Dazu wurden zunächst verschiedene Schulbücher der Oberstufe (Bader 2010; Bredthauer et al. 2007; Grehn 1992; Meyer und Schmidt 2003) miteinander verglichen, um die zentralen Themen und ihren Umfang zu erfassen. In einem nächsten Schritt wurden 16 Items aus dem TIMSS/III-Projekt (Baumert et al. 1998, 1999) ausgewählt, die geeignet waren entsprechende Themenbereiche abzudecken. Zur zusätzlichen Abdeckung der Themen der Oberstufe wurden vier weitere Items ergänzt. Das finale Instrument beinhal- tete schließlich 20 Multiple-Choice-Items, die die Bereiche Mechanik, Elektrizitätslehre und Magnetismus, Optik und Quantenphysik, Atom- und Kernphysik, Thermodynamik und die spezielle Relativitätstheorie umfassen. Durch die so beschriebene Konstruktion des Tests wird bereits eine gewisse Inhaltsvalidität gewährleistet.

Die Verteilung aller Studierenden $(N=239)$ sowie der Analysestichprobe $(N=158)$ über die mögliche Punktzahl ist in Abb. 3 dargestellt. Die Studierenden der Gesamtstichprobe erzielten im Mittel 9,81 $(S D=3,12)$ von 20 möglichen Punkten. Beim Vergleich der beiden Stichproben fällt auf, dass es bis auf den mittleren Bereich keine systematischen Unterschiede zwischen der Gesamtheit aller Studierenden und der Analysestichprobe gibt.

Zur Diskussion der Testgütekriterien werden die Daten aller Studierenden berücksichtigt. Die Abweichungen von der Normalverteilung liegen insgesamt im akzeptablen Bereich. Die Schwierigkeit der Aufgaben variiert von 0,22 bei Item 7 (Elektronen in einem homogenen elektrischen Feld) bis 0,90 bei Item 1 (Trägheitsprinzip) und deckt somit einen breiten Schwierigkeitsbereich ab. Vergleicht man die hier ermittelten klassischen Itemschwierigkeiten mit den Daten des TIMSS/III-Projekt, so fallen nur sehr geringe Abweichungen voneinander auf. Die mittlere Abweichung liegt bei den 16 Items bei $-0,02$, so dass sich die hier untersuchte Stichprobe bei den hier verwendeten Items nicht grundlegend von der TIMSS/III-Stichprobe unterscheidet. Bei der Betrachtung der Trennschärfen fällt jedoch auf, dass Item 18 negativ mit der Gesamtskala korreliert ist. Dieses Item wurde daher aus folgenden Analysen ausgeschlossen. Zur Untersuchung der Konstruktvalidität des Tests wurde im nächsten Schritt eine Raschskalierung vorgenommen (Bond und Fox 2007). Die Modellierung der Daten mit Hilfe des Rasch-Modells liefert dabei ebenfalls zufriedenstellende Item-Fit-Werte mit $0,90<$ WeightedMNSQ $<$ 1,12. Die berechnete EAP/PV- bzw. WLE-Reliabilität liegen mit 0,65 bzw. 0,63 noch in einem akzeptablen Bereich für einen Leistungstest mit relativ geringer Itemanzahl und breiter thematischer Ausrichtung. Der Constant-Wert von 0,05 deutet zudem auf eine gute Passung der mittleren Aufgabenschwierigkeit des Tests auf die mittlere Personenfähigkeit der Probanden hin (Neumann 2014). Die Standardabweichungen der Itemschwierigkeiten $\left(S D_{\text {Item }}=1,14\right)$ und der Personenfähigkeitsschätzer $\left(S D_{\text {Pers }}=0,90\right)$ liegen zudem in einer ähnlichen Größenordnung, so dass der Test das breite Fähigkeitsspektrum ausreichend abdeckt. Zur Verbesserung der Skalierung und damit der Erhöhung der Reliabilität wären ergänzende Items zur genaueren Charakterisierung der Mitte sowie Items für die Leistungsspitze mit mehr als 1,5 Logits nötig. Für die folgenden Auswertungen kann allerdings der WLE-Schätzer der Personenfähigkeit verwendet werden. 


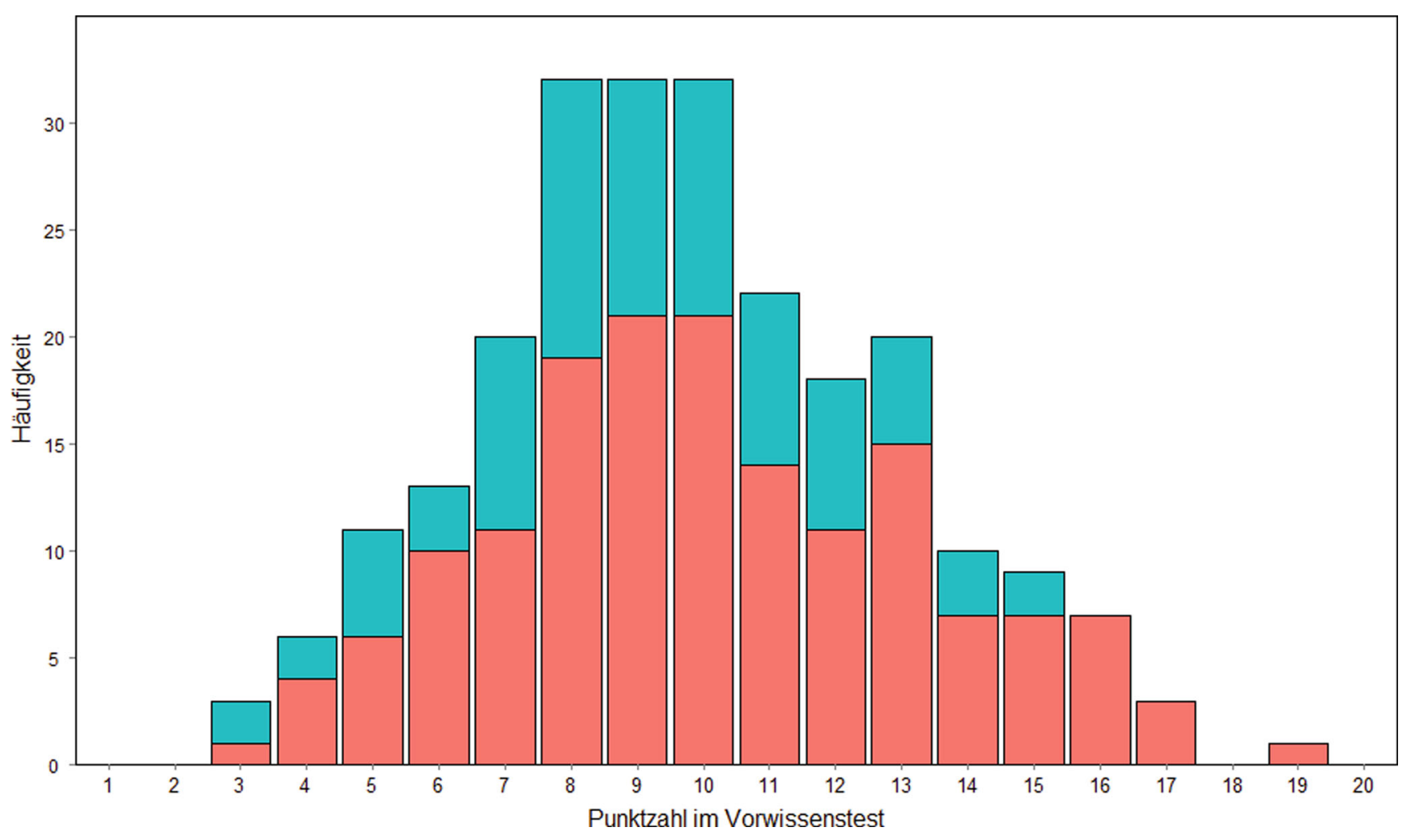

Abb. 3 Histogramm der erzielten Punktzahlen beim physikalischem Vorwissenstest der Studierenden, wobei der rote Anteil die Analysestichprobe $(N=158)$ umfasst und der blaue Anteil die zusätzlichen Probanden der Gesamtstichprobe $(N=239)$

Als abhängige Variable wurde das Abschneiden in der Abschlussklausur der Lehrveranstaltung betrachtet. Die Studierenden hatten die Möglichkeit, bereits kurz nach dem Ende der Vorlesungszeit oder am Ende der Semesterferien die Abschlussklausur zu absolvieren. Die Abschlussklausuren wurden vom Vorlesungsverantwortlichem erstellt und dienten der Überprüfung der Erreichung des vorgegebenen Lernziels. Dazu mussten die Studierenden innerhalb von 120 Minuten zehn Aufgaben beantworten, wobei die Lehramtsstudierenden der Physik zwischen der neunten und zehnten Aufgabe wählen konnten. Die Gesamtpunktzahl lag bei 50. Bei der Analyse der Aufgaben fällt auf, dass zunächst in einer ersten Aufgabe 10 Punkte durch das Erinnern und Anwenden von reinem Faktenwissen (Kratwohl 2010) zu erzielen waren. Dabei sollten bestimmte Beziehungen und Formeln wie beispielsweise die Galilei-Transformation, der Drehimpuls und der erste Hauptsatz der Thermodynamik angegeben werden. Die übrigen Aufgaben hingegen sind eher dem Aufgabentypus des Anwendens und Analysierens von konzeptuellem oder prozeduralem Wissens zuzuordnen. Dabei müssen die Studierenden zwar mathematische Modellierungsprozesse durchlaufen, allerdings steht eher die Konzeptanpassung (Trump und Borowski 2015) im Fokus. Im Vergleich mit den elf Übungsserien, die im Laufe des Semesters zu bearbeiten waren und die in den Übungsveranstaltung diskutiert wurden, sind große Übereinstimmungen zu den Klausuraufgaben festzustellen, so dass man aus dieser Perspektive von einer angemessenen Vorbereitung ausgehen kann. Es fällt allerdings auch auf, dass Aufgaben zur Thermodynamik nur einen sehr kleinen Teil im Spektrum der Klausuraufgaben ausmachen. Dies deckt sich allerdings wiederum damit, dass auch in den Übungsserien keine Aufgaben aus der Thermodynamik zu finden sind.

\section{Auswertung}

Die statistische Auswertung der Daten erfolgt in mehreren Schritten mit Hilfe der Statistiksoftware $R$. So sollen zunächst die deskriptiven Daten der Eingangsbefragung ausgewertet werden. Dabei soll der Fokus insbesondere auf Unterschiede in den kognitiven Eingangsmerkmalen zwischen den verschiedenen Studiengruppen gerichtet werden. Für die Auswertung der Abschlussklausur der Lehrveranstaltung an der Universität bieten sich prinzipiell zwei verschiedene Wege an. Zum einen wäre eine multiple Regression (Bortz 2005) zur Vorhersage der Klausurnote denkbar. Somit können dann Prädiktoren identifiziert werden, die ein möglichst gutes Abschneiden in der Prüfung begünstigen bzw. hemmen. Die zweite Auswertungsstrategie, die sich der logistischen Regression (Peng und So 2002) bedient, legt den Fokus weniger auf die Fragestellung nach 
einer möglichst guten Note, sondern eher auf Prädikatoren, die ein Bestehen und somit ein Fortführen des Studiums generell wahrscheinlicher machen. Diese Überlegungen entsprechen der Bewältigung der ,gatekeeper“-Kurse. Beide Verfahren sollen ihre Berücksichtigung in der Auswertung finden, um so ein möglichst umfassendes Bild zum Einfluss von Eingangsmerkmalen auf den Studienerfolg zu gewinnen. Die theoretisch vermuteten Prädiktoren werden zunächst einzeln auf ihre Vorhersagekraft mit Hilfe einer logistischen Regression analysiert. Dazu wird die Abschlussnote zunächst in eine dichotome Variable „Nicht-Bestehen/ Bestehen" umgewandelt.

Die logistische Regression ist ein Analyseverfahren, welches die Auftretenswahrscheinlichkeit einer abhängigen Variablen schätzt. Diese Schätzung beruht auf der MaximumLikelihood-Methode; ein Schätzverfahren also, welches die Wahrscheinlichkeit der auftretenden Daten unter dem parametrisierten Modell maximiert (Baltes-Götz 2012). Für ein derart spezifiziertes Schätzverfahren sollte die Stichprobengröße, einer Empfehlung von Backhaus et al. (2008) folgend, in jeder Kategorie der abhängigen Variable nicht kleiner als 25 sein, um zuverlässige Ergebnisse zu ermöglichen. Diese Voraussetzung ist für den vorliegenden Datensatz weitgehend erfüllt. Ein zentrales Gütekriterium für die lineare Regression ist der Anteil der erklärten Varianz $R^{2}$. Für die logistische Regression existieren solche allgemeingültigen Kriterien nicht. Dennoch ist es möglich, generelle Modellbewertungen durch den AIC oder die Analyse der Devianz vorzunehmen. Durch Differenzbildung der Devianz eines Modells mit der Devianz des Nullmodells (Likelihood-Ratio-Test) entsteht eine $\chi^{2}$-verteilte Prüfgröße, welche bei Signifikanz eine bessere „Passung“ des Modells indiziert (Peng und So 2002). Analog können auch zwei genestete Modelle bei Hinzunahme einzelner Prädiktoren auf eine Verbesserung des Modells überprüft werden, sofern die Stichprobe gleich bleibt. Es existieren weiterhin verschiedene deskriptive Maße, die eine ähnliche Funktion wie $R^{2}$ bei einer linearen Regression erfüllen sollen. Menard (2000) empfiehlt hierbei die Verwendung von McFaddens $R^{2}$ (manchmal auch als McFaddens Rho ${ }^{2}$ bezeichnet), da es konzeptionell dem gewöhnlichen $R^{2}$ gleicht und für den Vergleich verschiedener Modelle geeignet ist. Da es außerdem bei einer logistischen Regression um die Vorhersage der Auftretenswahrscheinlichkeit einer abhängigen Variable geht, können diese Wahrscheinlichkeiten genutzt werden um die korrekt klassifizierten Fälle anzugeben.

\section{Ergebnisse}

Zunächst werden die Ergebnisse der Eingangsbefragung vorgestellt. Dies soll vor allem einer Beschreibung der Stichprobe und der Besonderheiten der einzelnen Studien- fächer dienen. Mit Blick auf die Zielstellung der Untersuchung werden dann die Modelle der logistischen Regression vorgestellt. Dabei sollen zunächst die einzelnen möglichen Prädiktoren betrachtet und im Anschluss gemeinsam untersucht werden. Schließlich werden die Abschlussnoten der erfolgreichen Studierenden mit einer multiplen, linearen Regression analysiert.

Die Studierenden besitzen eine durchschnittliche Gesamtnote im Abitur von $M=2,38(S D=0,58)$. Dabei zeigen sich jedoch studiengangsspezifische Unterschiede, die in Abb. 4a dargestellt sind. Studierende des Bachelor Physik besitzen im Mittel die beste Abiturnote bei $M=2,23$ ( $S D=$ 0,60 ), während Lehramtsstudierende eine schlechtere $\mathrm{Ab}$ iturnote von $M=2,55(S D=0,42)$ aufweisen. Mit Hilfe des Levene-Tests konnte festgestellt werden, dass die Annahme der Gleichheit der Varianzen nicht zutreffend ist $(p<0,01)$. Aus diesem Grund wurde Welchs $F$-Test verwendet, um die Unterschiede zwischen den Gruppen auf Signifikanz zu prüfen. Dabei zeigte sich, dass die Unterschiede zwischen den Studiengängen in der Gesamtnote im Abitur signifikant sind (Welchs $F(2,95,94)=5,29, p<0,01)$. In einem nächsten Schritt wurden mit Hilfe des Games-Howell PostHoc-Tests die einzelnen Gruppen auf Unterschiede analysiert. Die Differenzen in den Abiturnoten der Studierenden des Bachelor Physik und der Lehramt Physik Studierenden erwiesen sich dabei als signifikant $(p<0,01, d=0,60)$.

Nach Ausschluss des Items mit negativer Trennschärfe beträgt der Mittelwert der untersuchten Studierenden $M=9,81(S D=3,33)$ von 19 möglichen Punkten im Test zum physikalischem Vorwissen, was einer mittleren Personenfähigkeit von 0,14 Logits entspricht. Auch hier lassen sich Gruppenunterschiede feststellen, die in Abb. 4b veranschaulicht sind. Hauptfachstudierende der Physik weisen die beste Leistung im Vorwissenstest mit $M=0,48$ Logits $(S D=1,07)$ auf. Studierende mit dem Ziel Bachelor Physik des Erdsystems zeigen den kleinsten Score mit durchschnittlich $M=-0,10(S D=0,87)$ Logits. Im Gegensatz zur Abiturnote ist hier von einer Varianzhomogenität zwischen den Gruppen auszugehen (Levene-Test, $p>0,05$ ). Die einfaktorielle Varianzanalyse verdeutlicht, dass die Studierendengruppen nicht dieselbe Leistung im Vorwissenstest zeigen (ANOVA: $F(2,155)=5,84, p<0,01)$. Der Einsatz des Tukey-Post-Hoc-Tests zeigt signifikante Unterschiede zwischen den Studierenden des Bachelor Physik mit den Gruppen Bachelor Physik des Erdsystems $(p<0,01, d=$ $0,59)$ und den Lehramtsstudierenden der Physik $(p=0,03$, $d=0,49$ ).

Von den 77 gemeldeten Abschlussklausuren haben 37 Studierende (48\%) die Klausur nicht bestanden und wurden mit der Note 5,0 bewertet. Bei den 40 bestandenen Klausuren variierten die Noten von 1,7 bis 4,0. Der Notendurchschnitt der Probanden des ersten Prüfungszeitpunktes lag bei $M=4,22(S D=1,01)$ und beim zweiten Prüfungs- 
zeitpunkt bei $M=4,45(S D=0,73)$. Beim Vergleich mit der Gesamtheit aller Studierenden fällt auf, dass es sich bereits um eine Positivselektion der Stichprobe bezüglich der Gesamtnote im Abitur handelt. So liegt der Mittelwert der Abiturnote der Klausurteilnehmenden mit $M=2,23$ ( $S D=$ 0,61) unter dem Mittelwert der Studierenden, die die Klausur nicht geschrieben haben oder ihre Klausurnote nicht angeben wollten von $M=2,52(S D=0,52, t(154)=-3,24$, $p<0,01)$.

Ausgehend von den theoretischen Vorüberlegungen wurden in einem nächsten Schritt die Modelle der logistischen Regression gebildet. Eine Übersicht über die verschiedenen Modelle ist in Tab. $1 \mathrm{zu}$ finden. Es fällt auf, dass durch die fehlenden Angaben in der Physiknote (s. oben) für einige Modelle nur Teilstichproben berücksichtigt werden konnten. Die Vergleiche zwischen den Modellen beziehen sich daher auf die jeweils übereinstimmenden Probanden. Die verschiedenen Prädiktoren wurden zunächst zur besseren Vergleichbarkeit standardisiert. Zunächst wurde der Effekt eines einzelnen Prädiktors auf die Wahrscheinlichkeit, eine Klausur zu bestehen, überprüft. In einem nächsten Schritt wurden die verschiedenen Prädiktoren gemeinsam betrachtet. In Modell 1 wurde zunächst nur die standardisierte Gesamtnote im Abitur als Prädiktor für die Wahrscheinlichkeit zum Bestehen der Abschlussklausur verwendet. Dieser erwies sich als signifikant $(\beta=-1,29, p<0,01)$. Verschlechtert sich also die Abiturnote um eine Standardabweichung, so steigt die Chance auf Misserfolg um $\frac{1}{0,27}=3,70$ an. Allein durch die Berücksichtigung der Abiturnote konnten $75 \%$ der Probanden korrekt auf die Ausprägungen Bestehen/Nicht-Bestehen zugeordnet werden. Ein ähnliches Ergebnis zeigt sich bei der alleinigen Untersuchung der letzten Note in Physik in Modell 2. Auch hier ist der Prädiktor signifikant $(\beta=1,85, p<0,01)$ und es konnten damit $70 \%$ der Probanden korrekt klassifiziert werden. Steigt der Wert der Physiknote um eine Standardabweichung, so nimmt die Chance zu Bestehen um das 6,36-fache im Vergleich mit einer durchschnittlichen Physiknote zu. Durch die Verwendung der Physiknote konnte ein McFadden $R^{2}$ von 0,60 erzielt werden, was bereits als ausgezeichnet anzusehen ist (Louviere et al. 2000). Schließlich ist auch der Prädiktor „Leistung im Vorwissenstest“ in Modell 3 signifikant zur Vorhersage des Bestehens der Abschlussklausur geeignet $(\beta=0,79, p<0,01)$. Bei einer um eine Standardabweichung verbesserten Leistung im Vorwissenstest nimmt die Chance auf das Bestehen um 2,20 zu. Insgesamt lassen sich mit diesem Prädiktor allein $62 \%$ der Fälle richtig zuordnen und das Modell erreicht ein McFadden $R^{2}$ von 0,09 , was auf
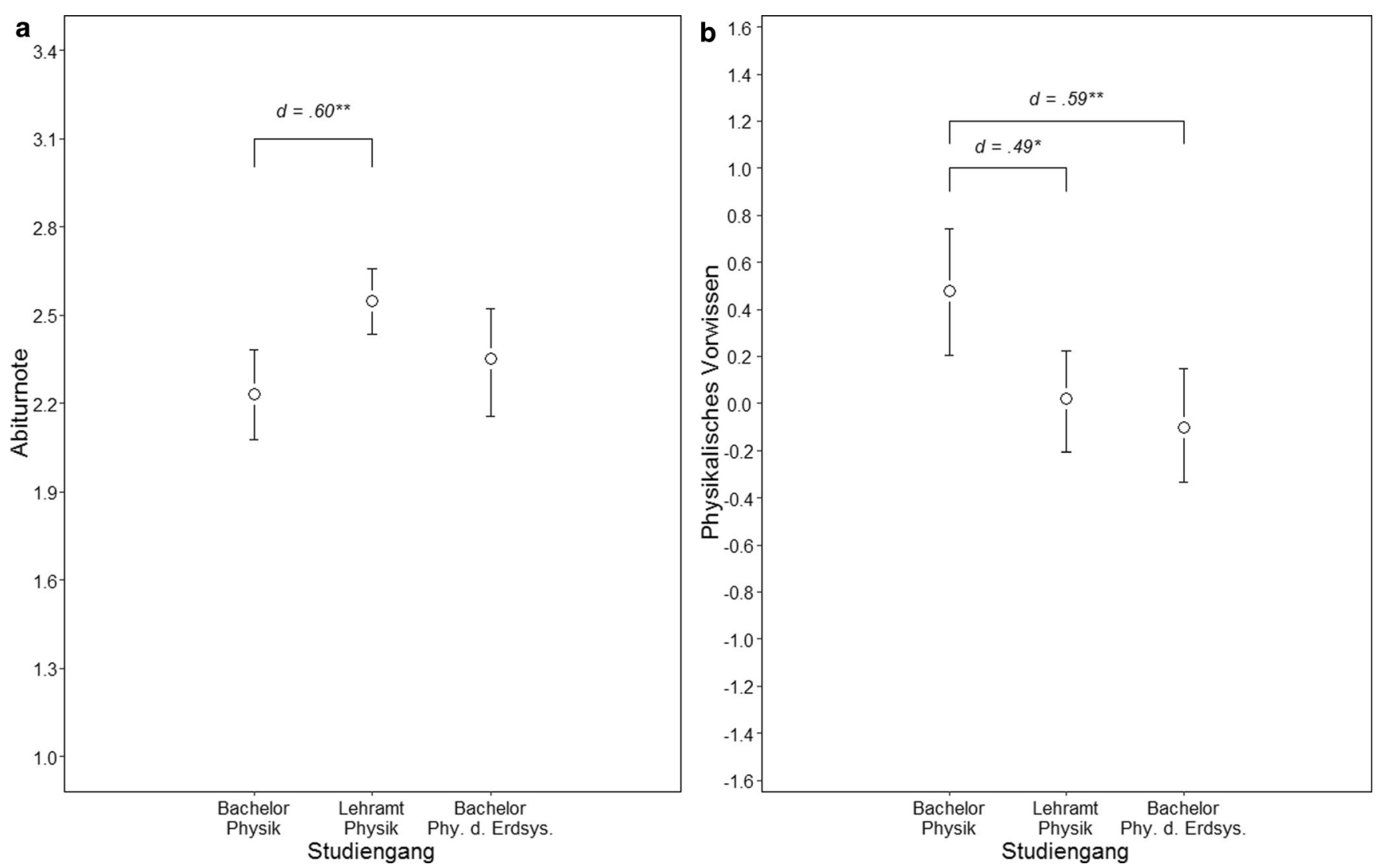

Abb. 4 Vergleich der kognitiven und fachlichen Voraussetzungen unter Angabe signifikanter Unterschiede (berechnet mit a Games-HowellPost-Hoc-Test b Tukey-Post-Hoc-Test; $\left.{ }^{*} p<0,01, * p<0,05\right)$ und des Standardfehlers a Gesamtnote im Abitur b Leistung im Vorwissenstest 
Tab. 1 Modelle der logistischen Regression mit der abhängigen Variablen „Bestehen der Abschlussklausur“

\begin{tabular}{|c|c|c|c|c|c|c|c|c|c|c|c|c|}
\hline & \multicolumn{2}{|c|}{ Modell 1} & \multicolumn{2}{|c|}{ Modell 2} & \multicolumn{2}{|c|}{ Modell 3} & \multicolumn{2}{|c|}{ Modell 4} & \multicolumn{2}{|c|}{ Modell 5} & \multicolumn{2}{|c|}{ Modell 6} \\
\hline & $\beta(\mathrm{SE})$ & $\begin{array}{l}\text { Odds } \\
\text { ratio }\end{array}$ & $\beta(\mathrm{SE})$ & $\begin{array}{l}\text { Odds } \\
\text { ratio }\end{array}$ & $\begin{array}{l}\beta \\
(\mathrm{SE})\end{array}$ & $\begin{array}{l}\text { Odds } \\
\text { ratio }\end{array}$ & $\begin{array}{l}\beta \\
(\mathrm{SE})\end{array}$ & $\begin{array}{l}\text { Odds } \\
\text { ratio }\end{array}$ & $\begin{array}{l}\beta \\
(\mathrm{SE})\end{array}$ & $\begin{array}{l}\text { Odds } \\
\text { ratio }\end{array}$ & $\beta(\mathrm{SE})$ & $\begin{array}{l}\text { Odds } \\
\text { ratio }\end{array}$ \\
\hline (Intercept) & $\begin{array}{l}0,09 \\
(0,27)\end{array}$ & 1,10 & $\begin{array}{l}0,28 \\
(0,38)\end{array}$ & 1,32 & $\begin{array}{l}0,09 \\
(0,24)\end{array}$ & 1,09 & $\begin{array}{l}0,45 \\
(0,47)\end{array}$ & 1,57 & $\begin{array}{l}0,14 \\
(0,28)\end{array}$ & 1,12 & $\begin{array}{l}0,46 \\
(0,47)\end{array}$ & 1,58 \\
\hline $\begin{array}{l}\text { Gesamtnote im } \\
\text { Abitur }\end{array}$ & $\begin{array}{l}-1,29 * * \\
(0,32)\end{array}$ & 0,27 & - & & - & & $\begin{array}{l}-1,77^{* *} \\
(0,67)\end{array}$ & 0,17 & $\begin{array}{l}-1,20^{* *} \\
(0,34)\end{array}$ & 0,30 & $\begin{array}{l}-1,79 * * \\
(0,67)\end{array}$ & 0,17 \\
\hline $\begin{array}{l}\text { Letzte Note in } \\
\text { Physik }\end{array}$ & - & & $\begin{array}{l}1,85^{* *} \\
(0,55)\end{array}$ & 6,36 & - & & $\begin{array}{l}1,85^{*} \\
(0,74)\end{array}$ & 6,39 & - & & $\begin{array}{l}2,02 * \\
(0,84)\end{array}$ & 7,56 \\
\hline $\begin{array}{l}\text { Leistung im } \\
\text { Vorwissenstest }\end{array}$ & - & & - & & $\begin{array}{l}0,79 * * \\
(0,28)\end{array}$ & 2,20 & - & & $\begin{array}{l}0,59 \\
(0,33)\end{array}$ & 1,80 & $\begin{array}{l}-0,37 \\
(0,70)\end{array}$ & 0,69 \\
\hline Probanden & 76 & & 46 & & 77 & & 46 & & 76 & & 46 & \\
\hline $\begin{array}{l}\text { Prozentzahl } \\
\text { korrekt zuge- } \\
\text { ordneter Fälle }\end{array}$ & $75 \%$ & & $70 \%$ & & $62 \%$ & & $87 \%$ & & $76 \%$ & & - & \\
\hline $\begin{array}{l}\text { Likelihood-Ratio- } \\
\text { Test }\end{array}$ & $\begin{array}{l}22,37 * * \\
\text { Verglich } \\
\text { Nullmod }\end{array}$ & mit & $\begin{array}{l}20,35^{* *} \\
\text { Verglic } \\
\text { Nullmo }\end{array}$ & mit & $\begin{array}{l}9,49 * * \\
\text { Verglic } \\
\text { Nullm }\end{array}$ & $\begin{array}{l}\text { en mit } \\
\text { el }\end{array}$ & $\begin{array}{l}10,72 * * \\
\text { Verglicl } \\
\text { Modell }\end{array}$ & n mit & $\begin{array}{l}3,51 \\
\text { Verglich } \\
\text { Modell } 1\end{array}$ & en mit & $\begin{array}{l}0,29 \\
\text { Verglich } \\
\text { Modell } 4\end{array}$ & mit \\
\hline AIC & 86,93 & & 47,07 & & 101,13 & & 38,22 & & 85,43 & & 39,93 & \\
\hline McFadden $R^{2}$ & 0,22 & & 0,60 & & 0,09 & & 0,70 & & 0,26 & & 0,70 & \\
\hline
\end{tabular}

Alle Prädiktoren standardisiert (Gesamtnote im Abitur auf Notenskala 1-6, Letzte Note in Physik auf Punkteskala 0-15)

$* * p \leq 0,01, * p \leq 0,05$

einen eher schlechten Modellfit hindeutet. Der LikelihoodRatio-Test verdeutlicht für alle drei Modelle eine bessere Passung auf die Stichprobe als das Nullmodell.

Im Modell 4 gingen sowohl die Gesamtnote im Abitur als auch die letzte Note in Physik gemeinsam in die Berechnung der logistischen Regression ein. Beide Prädiktoren erweisen sich hierbei als signifikant $\left(\beta_{\text {Abiturnote }}=-1,77, p<\right.$ $\left.0,01, \beta_{\text {Physiknote }}=1,85, p<0,05\right)$. Für die odds ratio bedeutet dieses Modell, dass bei einer durchschnittlichen Physiknote eine Änderung der Abiturnote um eine Standardabweichung die Misserfolgschance um $\frac{1}{0,17}=5,88$ steigt, wohingegen bei einer durchschnittlichen Abiturnote eine um eine Standardabweichung verbesserte Physiknote die Chance auf Erfolg um 6,39 erhöht. Durch die Berücksichtigung beider schulischen Merkmale konnten $87 \%$ der Probanden korrekt klassifiziert werden. McFaddens $R^{2}$ liegt bei 0,70 und der AIC nimmt mit 38,22 den kleinsten Wert aller Modelle an. Auch der Likelihood-Ratio-Test deutet auf einen besseren Modellfit im Vergleich mit Modell 1 hin $\left(\chi^{2}(1\right.$, $N=46)=10,72, p<0,01)$. Nutzt man statt der letzten Note in Physik die Leistung im Vorwissenstest als zusätzlichen Prädiktor neben der Gesamtnote im Abitur (Modell 5), so bleibt nur die Abiturnote signifikant $(\beta=-1,20, p<$ $0,01)$. Die Leistung im Vorwissenstest leistet hier also keinen inkrementellen Beitrag zur Verbesserung der Prognose des Bestehens, was auch der Likelihood-Ratio-Test im Vergleich zu Modell 1 suggeriert $\left(\chi^{2}(1, N=76)=3,51, p=\right.$ $0,06)$. Auch bei gleichzeitiger Berücksichtigung aller drei bisher verwendeter Prädiktoren im Modell 6 erweisen sich nur die Gesamtnote im Abitur und die letzte Note in Physik als signifikant. Es kommt zu keiner Verbesserung des Modellfits im Vergleich zu Modell $4\left(\chi^{2}(1, N=46)=0,29, p=\right.$ $0,59)$. Unter Berücksichtigung aller gegebener Kennwerte erweist sich also insgesamt das Modell 4, bei dem sowohl die Gesamtnote im Abitur als auch die letzte Note in Physik verwendet wurden, als aussagekräftigstes Modell. Mit diesem Modell ist es gelungen die Stichprobe sehr genau zu beschreiben.

In einem letzten Auswertungsschritt wurde der Einfluss der identifizierten Prädiktoren auf die Note der Abschlussklausur untersucht. Dazu wurden analog zu den Modellen der logistischen Regression multiple Regressionen berechnet, wobei nur Probanden betrachtet wurden, die die Klausur bestanden haben. Die Prädiktoren wurden dazu erneut am Mittelwert der nun zu betrachtenden Stichprobe $(N=40)$ standardisiert und schrittweise in die Regression eingegeben. Dabei bestätigte sich das zuvor gewonnene Bild, dass jeder der drei Prädiktoren einzeln signifikant bedeutsam für das Abschneiden in der Klausur ist. Bei der gleichzeitigen Berücksichtigung von zwei oder mehr Prädiktoren, wie es in Tab. 2 zusammengefasst ist, ergeben sich jedoch Unterschiede zu den Modellen der logistischen Regressionen. Bei einer durchschnittlichen Abiturnote und einer durchschnittlichen letzten Note in Physik ergibt sich eine Abschlussnote der Klausur von 3,40. Zur Verbesserung dieser Note trägt allerdings nur die Physiknote bei $(\beta=-0,24, p<0,05)$. Die Gesamtnote im Abitur ist nicht signifikant. Das gleiche Bild zeigt sich bei der simultanen Berücksichtigung von Abiturnote $(\beta=0,16, n$.s.) und Leistung im Vorwissenstest $(\beta=-0,24, p<0,05)$. Die Überprüfung des Einflusses 
Tab. 2 Modelle der linearen Regression mit der abhängigen Variablen „Note der Abschlussklausur“

\begin{tabular}{llll}
\hline & $\beta(\mathrm{SE})$ & $\beta(\mathrm{SE})$ & $\beta(\mathrm{SE})$ \\
\hline (Intercept) & $3,40(0,11)^{* *}$ & $3,35(0,10)^{* *}$ & $3,41(0,11)^{* *}$ \\
Gesamtnote im & $0,22(0,15)$ & $0,16(0,11)$ & $0,20(0,14)$ \\
Abitur & & & $-0,11(0,14)$ \\
Letzte Note in & $-0,27(0,12)^{*}$ & - & \\
$\begin{array}{l}\text { Physik } \\
\text { Leistung im }\end{array}$ & - & $-0,24(0,10)^{*}$ & $-0,27(0,12)^{*}$ \\
Vorwissenstest & & & \\
Probanden & 25 & 39 & 25 \\
$R^{2}$ & 0,33 & 0,25 & 0,45 \\
\hline
\end{tabular}

Alle Prädiktoren standardisiert (Gesamtnote im Abitur auf Notenskala 1-6, Letzte Note in Physik auf Punkteskala 0-15)

$* * p \leq 0,01 ; * p \leq 0,05$

aller drei Prädiktoren zeigt, dass lediglich die Leistung im Vorwissen seine Signifikanz wahrt $(\beta=-0,27, p<0,05)$. Bei einer durchschnittlichen Abiturnote und einer durchschnittlichen Physiknote führt also die Verbesserung der Leistung im Vorwissenstest um eine Standardabweichung zu einer um 0,27 verbesserten Abschlussnote in der Klausur der Einführungsveranstaltung. Dieses Modell liefert mit einem $R^{2}$ von 0,45 eine bedeutsame Aufklärung der Varianz in der Abschlussnote durch die drei Einflussfaktoren.

\section{Diskussion}

Ziel der vorliegenden Studie war es, die wesentlichen Aspekte der Studierfähigkeit zu identifizieren, die den Studienerfolg einer Einführungsveranstaltung im Fach Physik vorhersagen. Unter Studienerfolg wird in diesem Kontext die erfolgreiche Bewältigung der Einführungsveranstaltung in Form des Bestehens der Abschlussklausur und somit die Überwindung des ,gatekeeper“-Kurses (Gainen 1995) verstanden. Dies stellt einen bedeutsamen Schritt für eine insgesamt erfolgreiche Fortführung des Studiums dar. Die Untersuchung der Studierfähigkeit fokussiert auf die kognitive und fachliche Dimension, da sich diese in bisherigen Studien als besonders bedeutsam erwiesen haben (Konegen-Grenier 2002a). Dabei konnte die Bedeutsamkeit der Gesamtnote im Abitur als Maß für die kognitive Dimension der Studierfähigkeit für ein erfolgreiches Studium erneut bestätigt werden (Trapmann et al. 2007). Damit stehen die Ergebnisse aus der Physik in einer Linie mit Studien aus artverwandten Wissenschaften (Freyer et al. 2014, Rach und Heinze 2013). Insbesondere ist zu betonen, dass Studierende des Bachelor Physik an dieser Universität bereits bessere Abiturnoten in das Studium einbringen als Studierende des Lehramts. Diese Negativselektion für das Lehramtsstudium Physik relativiert die Erkenntnisse aus Studien, die Lehramtsstudierende generell (Neugebauer
2013) oder in den naturwissenschaftlichen Fächern (Roloff et al. 2015) untersuchten und keine Unterschiede in den Voraussetzungen im Vergleich mit Fachstudierenden feststellten. Allerdings berichten auch Albrecht und Nordmeier (2011) von besseren Voraussetzungen bei Fachstudierenden. Daraus lässt sich schlussfolgern, dass Aussagen über die Gesamtheit der Lehramtsstudierenden nur mit Vorsicht zu interpretieren sind. Vielmehr sind in den Untersuchungen an deutschen Hochschulen ebenfalls die Fachspezifik sowie universitätsspezifische Effekte zu berücksichtigen. Die spezifischen Curricula der einzelnen mathematisch-naturwissenschaftlichen Fächer und deren konkrete Ausgestaltung an einer Universität sowie die standortspezifische Studierendenzusammensetzung stellen hier wichtige Einflussgrößen dar. Auch in der Leistung im Vorwissenstest besitzen reine Fachstudierende Vorteile gegenüber Nebenfachstudierenden und Lehramtsstudierenden.

Der Effekt der Leistung im Vorwissenstest auf den Studienerfolg ist dabei weniger eindeutig. Die Wahrscheinlichkeit, die Abschlussklausur einer Einführungsveranstaltung in Physik im ersten Semester erfolgreich abzuschließen, wird dabei zwar bei der alleinigen Betrachtung der Leistung im Vorwissenstest beeinflusst, der Effekt ist jedoch im Vergleich mit den übrigen Prädiktoren gering und verschwindet sogar, wenn man die Abiturnote kontrolliert. Es gelingt somit einigen Studierenden mit einer adäquat ausgeprägten kognitiven Dimension der Studierfähigkeit ihre Defizite im physikalischen Vorwissen im Laufe des ersten Semesters so auszugleichen, dass ein Bestehen der Veranstaltung möglich wird. Erst bei genauerer Betrachtung der Studierenden, die die Klausur bestanden haben, wird deutlich, dass der Einfluss nicht gänzlich verschwindet. So wird die Leistung im Vorwissenstest als einziger Prädiktor signifikant für die Vorhersage der Abschlussnote. Zum Erreichen einer guten und sehr guten Note müssen die Studierenden der Taxonomie von Kratwohl (2010) folgend konzeptuelles oder prozedurales physikspezifisches Wissen anwenden und analysieren. Hierfür ist also eine breite und gut fundierte Wissensbasis aus der Schulphysik hilfreich. Das Ergebnis in dieser Studie unterscheidet sich somit von den Ergebnissen in Mathematik (Rach \& Heinze, 214) und Chemie (Freyer et al. 2014) für die Bedeutung des Vorwissens. Dabei ist jedoch zu beachten, dass die Vorwissensitems in den zitierten Untersuchungen viel stärker auf die Inhaltsbereiche der Vorlesung (Anorganische Chemie und Analysis) konzipiert waren als dies in der vorliegenden Studie der Fall war. Dem theoretischen Modell (Heldmann 1984; KonegenGrenier 2002a) folgend, wird in der vorliegenden Untersuchung unter den fachlichen Voraussetzungen die gesamte Bandbreite der Schulphysik verstanden und nicht bereits auf die Mechanik und Thermodynamik fokussiert.

Der Effekt der letzten Note in Physik auf den Studienerfolg stellte sich in dieser Studie als überraschend groß her- 
aus. Vergleicht man die Effekte von Abiturnote und Physiknote, so liegen beide in einer ähnlichen Größenordnung bzw. hat die Physiknote einen leicht größeren Einfluss auf die Überwindung des ,gatekeeper“. Dies steht zunächst im Gegensatz zu bisherigen Studien (Trapmann et al. 2007), allerdings lassen sich auch für die letzte Physiknote ähnliche Argumente wie für die Wirksamkeit der Abiturnote für den Studienerfolg anführen. Die Physiknote umfasst neben einer reinen Fachwissenskomponente auch die Fähigkeit in Tests und Klausuren positive Ergebnisse zu erzielen, was sich auch in der geringen Korrelation von Physiknote und Leistung im Vorwissenstest von $r=0,35(p<0,01, N=91)$ in der Analysestichprobe zeigt. Einschränkend muss jedoch angemerkt werden, dass nur ein Teil der Studierenden ihre Physiknote angegeben haben, so dass keine Aussagen über alle Studierenden der Einführungsveranstaltung möglich sind. Insgesamt bestätigen die Befunde die Ergebnisse der Studie von Konegen-Grenier (2002a), dass die kognitive und fachliche Dimension der Studierfähigkeit für den Studienerfolg besonders bedeutsam ist.

Ausgehend von theoretischen Überlegungen wurde in der vorliegenden Studie der Fokus auf kognitive und fachliche Prädiktoren für den Studienerfolg gelegt. Für die persönliche Voraussetzung beim Studienwahlmotiv konnten keine Zusammenhänge mit dem Studienerfolg gefunden werden. Es wäre jedoch denkbar, dass nicht die Motivation für die Wahl des Studienfachs einen Einfluss auf den Studienerfolg hat, sondern Faktoren, die erst im Laufe des Semesters durch den Kontakt mit dem Studium wirksam werden. Studierende werden durch den Übergang von der Schule zur Hochschule mit einer neuen Lernumgebung konfrontiert (Gueudet 2008). Dabei müssen sie ihre bisher verwendeten Lernstrategien adaptieren und auch lernen mit negativen Erlebnissen umzugehen. Eine Schlüsselfähigkeit könnte demnach der Umgang mit Rückschlägen sein, was durch die Fähigkeit der ,academic buoyancy“ (Neumann et al. 2015, 2016; Martin und Marsh 2008) charakterisiert ist. Für die zukünftige Forschung im Bereich des Studienerfolgs im Fach Physik ist es also bedeutsam, neben den kognitiven und fachspezifischen Eingangsmerkmalen auch die Entwicklung motivationaler und volitionaler Faktoren in den Blick zu nehmen. Dabei ist jedoch zu beachten, dass Studierende durch ihre Studienwahl bereits eine gewisse Motivation mitbringen, so dass eingesetzte Instrumente insbesondere im oberen Bereich der Skala sensitiv sein sollten. Bei der Interpretation der Studie muss weiterhin darauf geachtet werden, dass sich die Befragung auf einen Universitätsstandort in Deutschland beschränkt. Dies hat allerdings den Vorteil, dass die Treatment-Bedingungen - hier also die Vorlesung - für alle Teilnehmer konstant gehalten wurden und somit der Einfluss von Störvariablen reduziert ist. Um die Ergebnisse dennoch weiter generalisieren zu können, sollten ähnliche Untersuchungen an weiteren
Hochschulen in Deutschland durchgeführt werden. Sollten sich die Effekte für unterschiedliche Vorlesende oder unter Berücksichtigung einer Mehrebenenstruktur bestätigen, könnten stärkere Generalisierungen getroffen werden. Die Arbeiten von Albrecht und Nordmeier (2011) und dieser Beitrag stellen dafür einen ersten Schritt dar.

Insgesamt konnte in der Studie die Bedeutung der kognitiven und fachlichen Dimension der Studierfähigkeit für die Prognose des Studienerfolgs bestätigt werden. Es konnte ebenfalls gezeigt werden, dass nicht-vorhandenes Vorwissen zum Teil im Laufe eines Semesters durch die Vorlesung kompensiert werden kann. Für die Praxis an den Hochschulen ist es also nötig, die vorhandenen Sicherungsnetze dahingehend auszubauen, dass nicht nur die fachliche Dimension der Studierfähigkeit gestärkt wird, sondern vor allem die kognitive Dimension Unterstützung findet. Durch die Förderung analytischer Fähigkeit sowie dem Abstraktionsund Differenzierungsvermögens könnten Studierende, die sowohl schlechte Abiturnoten als auch Probleme im fachlichen Bereich haben, befähigt werden, den ,gatekeeper“ Einführungsveranstaltung zu überwinden. Weiterhin ist es notwendig, verstärkt Anreize zu schaffen ein Lehramtsstudium aufzunehmen, um so geeignete Kandidaten zu rekrutieren, die erfolgreich in ihrem Studium und damit später im Klassenzimmer sein können. Nur durch die optimale Förderung möglichst vieler Studierender kann dem Nachwuchsproblem in den Naturwissenschaften besser begegnet werden.

Open Access Dieser Artikel wird unter der Creative Commons Namensnennung 4.0 International Lizenz (http://creativecommons.org/ licenses/by/4.0/deed.de) veröffentlicht, welche die Nutzung, Vervielfältigung, Bearbeitung, Verbreitung und Wiedergabe in jeglichem Medium und Format erlaubt, sofern Sie den/die ursprünglichen Autor(en) und die Quelle ordnungsgemäß nennen, einen Link zur Creative Commons Lizenz beifügen und angeben, ob Änderungen vorgenommen wurden.

\section{Literatur}

Albrecht, A., \& Nordmeier, V. (2011). Ursachen des Studienabbruchs in Physik. Eine explorative Studie. Die Hochschule, 20(2), 131-145.

Angell, C., Guttersrud, O., Henriksen, E. K., \& Isnes, A. (2004). Physics: frightful, but fun. pupils' and teachers' views of physics and physics teaching. Science Education, 88(5), 683-706.

Anger, C., Koppel, O., \& Plünnecke, A. (2014). MINT-Herbstreport 2014. MINT - Attraktive Perspektiven und demografische Herausforderung. Gutachten für BDA, BDI, MINT Zukunft schaffen und Gesamtmetall.

Backhaus, K., Erichson, B., Plinke, W., \& Weiber, R. (2008). Multivariate Analysemethoden. Berlin: Springer.

Bader, F. (Hrsg.). (2010). PHYSIK Gymnasium SEK II. Braunschweig: Schroedel.

Baltes-Götz, B. (2012). Logistische Regressionsanalyse mit SPSS. Trier: Universität Trier.

Baumert, J., Bos, W., \& Watermann, R. (1998). TIMSS/III: Schülerleistungen in Mathematik und den Naturwissenschaften am Ende 
der Sekundarstufe II im internationalen Vergleich. Berlin: MaxPlanck-Institut für Bildungsforschung.

Baumert, J., Bos, W., Klieme, E., Lehmann, R., Lehrke, M., Hosenfeld, I., Neubrand, J., \& Watermann, R. (Hrsg.). (1999). Testaufgaben zи TIMSS/III. Mathematisch-naturwissenschaftliche Grundbildung und voruniversitäre Mathematik und Physik der Abschlußklassen der Sekundarstufe II (Population 3). Berlin: Max-PlanckInstitut für Bildungsforschung.

Blömeke, S. (2009). Ausbildungs- und Berufserfolg im Lehramtsstudium im Vergleich zum Diplom-Studium. Zur prognostischen Validität kognitiver und psycho-motivationaler Auswahlkriterien. Zeitschrift für Erziehungswissenschaften, 12(1), 82-110.

Bond, T. G., \& Fox, C.M. (2007). Applying the Rasch model. Fundamental measurements in the human sciences (2. Aufl.). Mahwah: Lawrence Erlbaum Associates.

Bortz, J. (2005). Statistik für Human- und Sozialwissenschaftler. Heidelberg: Springer.

Bredthauer, W., Bruns, K.G., Dorn, H.-J., Grote, M., Hannibal, L., Renner, M., Schell, N., Schmidt, M., Wojke, P., \& Zimmerscheid, F. (2007). Impulse Physik Oberstufe. Stuttgart: Klett.

Deutsche Physikalische Gesellschaft (2014). Zur fachlichen und fachdidaktischen Ausbildung für das Lehramt Physik. Bad Honnef: Deutsche Physikalische Gesellschaft.

Freyer, K., Epple, M., Brand, M., Schiebener, J., \& Sumfleth, E. (2014). Studienerfolgsprognose bei Erstsemesterstudierenden der Chemie. Zeitschrift für Didaktik der Naturwissenschaften, 20(1), 129-142.

Gainen, J. (1995). Barriers to success in quantitative gatekeeper courses. In J. Gainen \& E.W. Williamsen (Hrsg.), Fostering student success in quantitative gateway courses $\mathrm{S}$, (Bd. 61, S. 5-14). San Francisco: Jossey-Bass.

Grehn, J. (Hrsg.). (1992). Metzler Physik. Hannover: Metzler.

Gueudet, G. (2008). Investigating the secondary-tertiary transition. Educational Studies in Mathematics, 67, 237-254.

Hattie, J. A.C. (2009). Visible learning. A synthesis of over 800 Metaanalyses relating to achievement. London, New York: Routledge.

Hazari, Z., Tai, R.H., \& Sadler, P.M. (2007). Gender differences in introductory university physics performance. The influence of high school preparation and affective factors. Science Education, 91(6), 847-876.

Heldmann, W. (1984). Studierfähigkeit. Ergebnis einer Umfrage. Göttingen: Schwartz.

Heldmann, W., \& Finkenstaedt, T. (1998). Voraussetzungen und Rahmenbedingungen des Hochschulstudiums. Bad Honnef: K. H. Bock.

Heublein, U., Hutzsch, C., Schreiber, J., Sommer, D., \& Besuch, G. (2010). Ursachen des Studienabbruchs in Bachelor- und in herkömmlichen Studiengängen. Ergebnisse einer bundesweiten Befragung von Exmatrikulierten des Studienjahres 2007/08 (HIS:Forum Hochschule 2/2010). Hannover: HIS.

Heublein, U., Richter, J., Schmelzer, R., \& Sommer, D. (2014). Die Entwicklung der Studienabbruchquoten an den deutschen Hochschulen. Statistische Berechnungen auf der Basis des Absolventenjahrgangs 2012. Hannover: Deutsches Zentrum für Hochschul- und Wissenschaftsforschung (DZHW).

Kazemzadeh, F., Minks, K.-H., \& Nigmann, R.-R. (1987). „Studierfähigkeit" - eine Untersuchung des Übergangs vom Gymnasium zur Universität. Hannover: HIS.

Kirsch, B., \& Vo Thi Anh, T. (1996). Problemerleben und Problembewältigung beim Übergang von der Schule zur Hochschule. In J. Lompscher \& H. Mandl (Hrsg.), Lehr- und Lernprobleme im Studium (S. 185-206). Bern: Huber.

Klemm, K. (2015). Lehrerinnen und Lehrer der MINT-Fächer: Zur Bedarfs- und Angebotsentwicklung in den allgemein bildenden Schulen der Sekundarstufen I und II am Beispiel Nordrhein-Westfalens. Gutachten im Auftrag der Deutsche Telekom Stiftung
Köller, O. (2013). Abitur und Studierfähigkeit. In J. Asdonk, S. U. Kuhnen \& P. Bornkessel (Hrsg.), Von der Schule zur Hochschule (S. 25-49). Münster: Waxmann.

Konegen-Grenier, C. (2002a). Studierfähigkeit und Hochschulzugang. Köln: Deutscher Instituts-Verlag.

Konegen-Grenier, C. (2002b). Studierfähigkeit und Hochschulzugang. Forschung \& Lehre, 9, 481-483.

Kratwohl, D. R. (2010). A revision of bloom's taxonomy. An overview. Theory Into Practice, 41(4), 212-218.

Louviere, J. J., Hensher, D. A., \& Swait, J. D. (2000). Stated choice methods. Analysis and application. Cambridge: Cambridge University Press.

Martin, A. J., \& Marsh, H. W. (2008). Academic buoyancy: towards an understanding of student's everyday academic resilience. Journal of School Psychology, 46, 53-83.

Matzdorf, R., \& Düchs, G. (2013). Immer mehr Parkstudierende. Statistiken zum Physikstudium an den Universitäten in Deutschland 2013. Physik Journal, 12(8/9), 29-33.

Menard, S. (2000). Coefficients of determination for multiple logistic regression analysis. The American Statistician, 54, 17-24.

Meyer, L., \& Schmidt, G.-D. (Hrsg.). (2003). Physik Gymnasiale Oberstufe. Berlin: Duden Paetec.

Neugebauer, M. (2013). Wer entscheidet sich für ein Lehramtsstudium - und warum? Eine empirische Überprüfung der These von der Negativselektion in den Lehrerberuf. Zeitschrift für Erziehungswissenschaften, 16(1), 157-184.

Neumann, K. (2014). Rasch-Analyse naturwissenschaftsbezogener Leistungstests. In D. Krüger, I. Parchmann, \& H. Schecker (Hrsg.), Methoden der naturwissenschaftsdidaktischen Forschung (S. 355-370). Berlin, Heidelberg: Springer.

Neumann, I., Jeschke, C., \& Heinze, A. (2015). Transition school university: measuring mathematics freshmen's academic buoyan$c y$. Meeting of the International Group for the Psychology of Mathematics Education (PME39), Hobart, 13.-18. Jul 2015.

Neumann, I., Sorge, S., Jeschke, C., Heinze, A., \& Neumann, K. (2016). Zur Academic Buoyancy von Physikstudierenden. In C. Maurer (Hrsg.), Authentizität und Lernen - das Fach in der Fachdidaktik (S. 86-88). Gesellschaft für Didaktik der Chemie und Physik, Jahrestagung in Berlin 2015: Universität Regensburg.

Peng, C.-Y.J., \& So, T.-S.H. (2002). Logistic regression analysis and reporting: a primer. Understanding Statistics, 1(1), 31-70.

Rach, S., \& Heinze, A. (2013). Welche Studierenden sind im ersten Semester erfolgreich? Zur Rolle von Selbsterklärungen beim Mathematiklernen in der Eingangsphase. Journal für MathematikDidaktik, 34, 121-147.

Rach, S., \& Heinze, A. (2014). Individuelle Bedingungsfaktoren für den Studienerfolg im ersten Semester im Mathematikstudium. In J. Roth \& J. Ames (Hrsg.), Beiträge zum Mathematikunterricht 2014 (S. 935-938). Münster: WTM.

Rindermann, H., \& Oubaid, V. (1999). Auswahl von Studienanfängern durch Universitäten - Kriterien, Verfahren und Prognostizierbarkeit des Studienerfolgs. Zeitschrift für Differentielle und Diagnostische Psychologie, 20(3), 172-191.

Roloff, H. J., Klusmann, U., Lüdtke, O., \& Trautwein, U. (2015). Who becomes a teacher?. Challenging the „negative selection“ hypothesis. Learning and Instruction, 36, 46-56.

Schiefele, U., \& Urhahne, D. (2000). Motivationale und volitionale Bedingungen der Studienleistung. In U. Schiefele \& K.-P. Wild (Hrsg.), Interesse und Lernmotivation. Untersuchungen zur Entwicklung, Förderung und Wirkung (S. 183-205). Münster: Waxmann.

Schiefele, U., Streblow, L., Ermgassen, U., \& Moschner, B. (2003). Lernmotivation und Lernstrategien als Bedingungen der Studienleistung. Ergebnisse einer Längsschnittstudie. Zeitschrift für Pädagogische Psychologie, 17(3/4), 185-198. doi:10.1024//10100652.17.3.185.

Schiefele, U., Streblow, L., \& Brinkmann, J. (2007). Aussteigen oder Durchhalten. Was unterscheidet Studienabbrecher von anderen 
Studierenden? Zeitschrift für Entwicklungspsychologie und Pädagogische Psychologie, 39(3), 127-140.

Shaw, E. J., \& Barbuti, S. (2010). Patterns of Persistence in Intended College Major with a Focus on STEM Majors. NACADA. Journal, 30(2), 19-34.

Statistisches Bundesamt (2015). Bildung und Kultur. Studierende an Hochschulen - Vorbericht - Wintersemester 2014/2015, (Fachserie 11, Reihe 4.1). Wiesbaden: Statistisches Bundesamt.

Thiel, F., Veit, S., Blüthmann, I., Lepa, S., \& Ficzko, M. (2008). Ergebnisse der Befragung der Studierenden in den Bachelorstudiengängen an der Freien Universität. Sommersemester, Bd. 2008. Berlin: Freie Universität Berlin.

Trapmann, S., Hell, B., Weigand, S., \& Schuler, H. (2007). Die Validität von Schulnoten zur Vorhersage des Studienerfols - eine Metaanalyse. Zeitschrift für Pädagogische Psychologie, 21(1), 11-27.
Trautwein, U., \& Lüdtke, O. (2004). Aspekte von Wissenschaftspropädeutik und Studierfähigkeit. In O. Köller, R. Watermann, U. Trautwein \& O. Lüdtke (Hrsg.), Wege zur Hochschulreife in Baden-Würtenberg. TOSCA - Eine Untersuchung an allgemeinbildenden und beruflichen Gymnasien (S. 327-367). Opladen: Leske + Budrich.

Trump, S., \& Borowski, A. (2015). Mathematik in der Physik der Sekundarstufe II - Wie und Welche? In S. Bernholt (Hrsg.), Heterogenität und Diversität - Vielfalt der Voraussetzungen im naturwissenschaftlichen Unterricht. Gesellschaft für Didaktik der Chemie und Physik, Jahrestagung in Bremen, (Bd. 2014, S. 370-372). Kiel: IPN.

Wissenschaftsrat (2000). Empfehlungen zur Einführung neuer Studienstrukturen und-abschlüsse in Deutschland. Berlin. 